\title{
Emulsion Science and Technology: A General Introduction
}

Tharwat F. Tadros

\section{1}

\section{Introduction}

Emulsions are a class of disperse systems consisting of two immiscibleliquids [1-3]. The liquid droplets (the disperse phase) are dispersed in a liquid medium (the continuous phase). Several classes of emulsion may be distinguished, namely oil-in-water $(O / W)$, water-in-oil (W/O) and oil-in-oil (O/O). The latter class may be exemplified by an emulsion consisting of a polar oil (e.g. propylene glycol) dispersed in a nonpolar oil (paraffinic oil), and vice versa. In order to disperse two immiscible liquids a third component is required, namely the emulsifier; the choice of emulsifier is crucial not only for the formation of the emulsion but also for its long-term stability [1-3].

Emulsions may be classified according to the nature of the emulsifier or the structure of the system (see Table 1.1).

Several processes relating to the breakdown of emulsions may occur on storage, depending on:

- the particle size distribution and the density difference between the droplets and the medium;

- the magnitude of the attractive versus repulsive forces, which determines flocculation;

- the solubility of the disperse droplets and the particle size distribution, which in turn determines Ostwald ripening;

- the stability of theliquid film between the droplets, which determines coalescence; and

- phase inversion.

The various breakdown processes are illustrated schematically in Figure 1.1.

The physical phenomena involved in each breakdown process is not simple, and requires an analysis to be made of the various surface forces involved. In addition, the above processes may take place simultaneously rather then consecutively, which in turn complicates the analysis. Model emulsions, with monodisperse droplets, cannot be easily produced and hence any theoretical treatment must take into account the effect of 
Table 1.1 Classification of emulsion types.

Nature of emulsifier

Simple molecules and ions

Nonionic surfactants

Surfactant mixtures

Ionic surfactants

Nonionic polymers

Polyelectrolytes

Mixed polymers and surfactants

Liquid crystalline phases

Solid particles

\section{Structure of the system}

Nature of internal and external phase:

$\mathrm{O} / \mathrm{W}, \mathrm{W} / \mathrm{O}$

Micellar emulsions (microemulsions)

Macroemulsions

Bilayer droplets

Double and multiple emulsions

Mixed emulsions

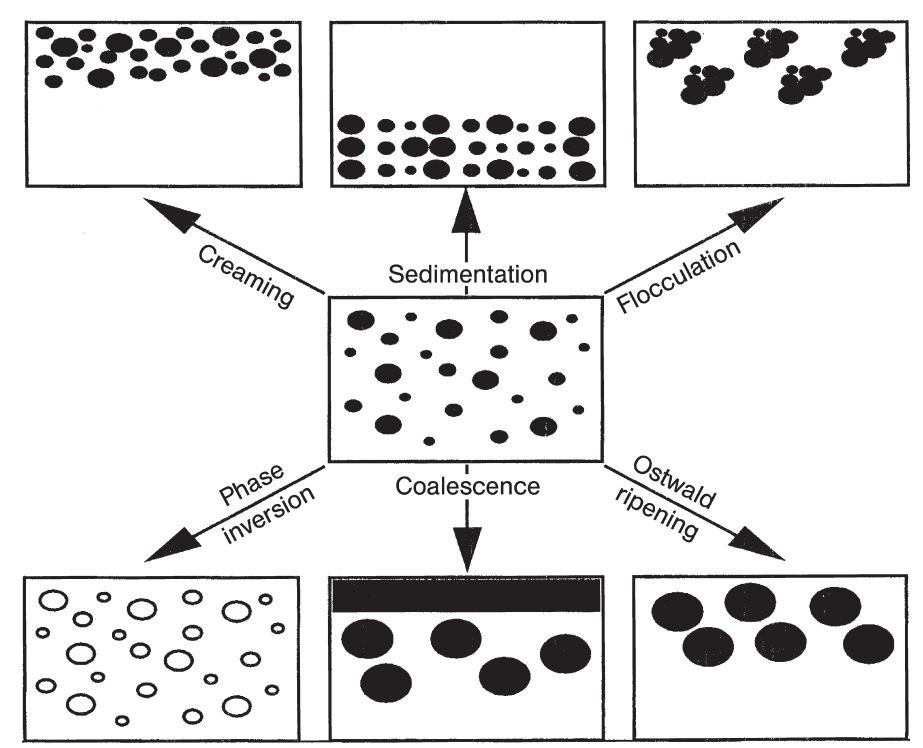

Figure 1.1 Schematic representation of the various breakdown processes in emulsions.

droplet size distribution. Theories that take into account the polydispersity of the system are complex, and in many cases only numerical solutions are possible. In addition, the measurement of surfactant and polymer adsorption in an emulsion is not simple, and such information must be extracted from measurements made at a planar interface.

A summary of each of the above breakdown processes is provided in the following sections, together with details of each process and methods for its prevention.

Creaming and Sedimentation This process results from external forces, usually gravitational or centrifugal. When such forces exceed the thermal motion of the droplets (Brownian motion), a concentration gradient builds up in the system such 
that the larger droplets move more rapidly either to the top (if their density is less than that of the medium) or to the bottom (if their density is greater than that of the medium) of the container. In the limiting cases, the droplets may form a close-packed (random or ordered) array at the top or bottom of the system, with the remainder of the volume occupied by the continuous liquid phase.

Flocculation This process refers to aggregation of the droplets (without any change in primary droplet size) into larger units. It is the result of the van der Waals attractions which are universal with all disperse systems. Flocculation occurs when there is not sufficient repulsion to keep the droplets apart at distances where the van der Waals attraction is weak. Flocculation may be either 'strong' or 'weak', depending on the magnitude of the attractive energy involved.

Ostwald Ripening (Disproportionation) This effect results from the finite solubility (etc.) of the liquid phases. Liquids which are referred to as being 'immiscible' often have mutual solubilities which are not negligible. With emulsions which are usually polydisperse, the smaller droplets will have a greater solubility when compared to larger droplets (due to curvature effects). With time, the smaller droplets disappear and their molecules diffuse to the bulk and become deposited on the larger droplets. With time, the droplet size distribution shifts to larger values.

Coalescence This refers to the process of thinning and disruption of the liquid film between the droplets, with the result that fusion of two or more droplets occurs to form larger droplets. The limiting case for coalescence is the complete separation of the emulsion into two distinct liquid phases. The driving force for coalescence is the surface or film fluctuations; this results in a close approach of the droplets whereby the van der Waals forces are strong and prevent their separation.

Phase Inversion This refers to the process whereby there will be an exchange between the disperse phase and the medium. For example, an O/W emulsion may with time or change of conditions invert to a W/O emulsion. In many cases, phase inversion passes through a transition state whereby multiple emulsions are produced.

\section{2}

\section{Industrial Applications of Emulsions}

Several industrial systems consist of emulsions of which the following are worthy of mention:

- Food emulsions, such as mayonnaise, salad creams, deserts and beverages.

- Personal care and cosmetic products, such as hand-creams, lotions, hair-sprays and sunscreens.

- Agrochemicals - self-emulsifiable oils which produce emulsions on dilution with water, emulsion concentrates (droplets dispersed in water; EWs) and crop oil sprays. 
- Pharmaceuticals, such as anesthetics of O/W emulsions, lipid emulsions, double and multiple emulsions.

- Paints, such as emulsions of alkyd resins and latex emulsions.

- Dry-cleaning formulations; these may contain water droplets emulsified in the drycleaning oil, which is necessary to remove soils and clays.

- Bitumen emulsions are prepared stable in their containers but, when applied the road chippings, they must coalesce to form a uniform film of bitumen.

- Emulsions in the oil industry - many crude oils contain water droplets (e.g. North Sea oil); these must be removed by coalescence followed by separation.

- Oil slick dispersants - oil spilled from tankers must be emulsified and then separated. The emulsification of unwanted oil is a very important process in pollution control.

The above-described utilization of emulsions in industrial processes justifies the vast amount of basic research which is conducted aimed at understanding the origins of the instability of emulsions and developing methods to prevent their break down. Unfortunately, fundamental research into emulsions is not straightforward, as model systems (e.g. with monodisperse droplets) are difficult to produce. In fact, in many cases, the theoretical bases of emulsion stability are not exact and consequently semi-empirical approaches are used.

\section{3}

\section{The Physical Chemistry of Emulsion Systems}

\subsection{1}

\section{The Interface (Gibbs Dividing Line)}

An interface between two bulk phases, such as liquid and air (or liquid/vapor) or two immiscible liquids (oil/water), may be defined provided that a dividing line is introduced (Figure 1.2). The interfacial region is not a layer that is one molecule thick; rather, it has a thickness $\delta$ with properties that differ from those of the two bulk phases $\alpha$ and $\beta$.

By using the Gibbs model, it is possible to obtain a definition of the surface or interfacial tension $\gamma$.

The surface free energy $d G^{\sigma}$ is composed of three components: an entropy term $S^{\sigma} d T$; an interfacial energy term $A d \gamma$; and a composition term $\sum n_{i} d \mu_{i}$ (where $n_{i}$ is the

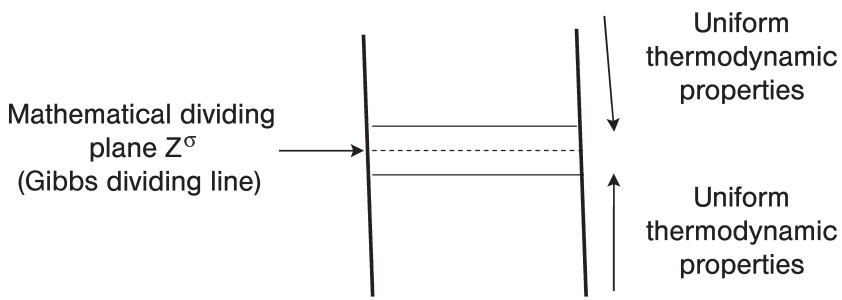

Figure 1.2 The Gibbs dividing line. 
number of moles of component $i$ with chemical potential $\mu_{i}$ ). The Gibbs-Deuhem equation is therefore,

$$
d G^{\sigma}=-S^{\sigma} d T+A d \gamma+\sum n_{i} d \mu_{i}
$$

At constant temperature and composition,

$$
\begin{gathered}
d G^{\sigma}=A d \gamma \\
\gamma=\left(\frac{\partial G^{\sigma}}{\partial A}\right)_{T, n_{i}}
\end{gathered}
$$

For a stable interface $\gamma$ is positive - that is, if the interfacial area increases, then $G^{\sigma}$ increases. Note that $\gamma$ is energy per unit area $\left(\mathrm{mJ} \mathrm{m}^{-2}\right)$, which is dimensionally equivalent to force per unit length $\left(\mathrm{mN} \mathrm{m}^{-1}\right)$, the unit usually used to define surface or interfacial tension.

For a curved interface, one should consider the effect of the radius of curvature. Fortunately, $\gamma$ for a curved interface is estimated to be very close to that of a planar surface, unless the droplets are very small $(<10 \mathrm{~nm})$. Curved interfaces produce some other important physical phenomena which affect emulsion properties, such as the Laplace pressure $\Delta p$ which is determined by the radii of curvature of the droplets,

$$
\Delta p=\gamma\left(\frac{1}{r_{1}}+\frac{1}{r_{2}}\right)
$$

where $r_{1}$ and $r_{2}$ are the two principal radii of curvature.

For a perfectly spherical droplet $r_{1}=r_{2}=r$ and

$$
\Delta p=\frac{2 \gamma}{r}
$$

For a hydrocarbon droplet with radius $100 \mathrm{~nm}$, and $\gamma=50 \mathrm{mN} \mathrm{m}^{-1}, \Delta p \sim 10^{6} \mathrm{~Pa}$ (10 atm).

\section{4}

\section{The Thermodynamics of Emulsion Formation and Breakdown}

Consider a system in which an oil is represented by a large drop 2 of area $A_{1}$ immersed in a liquid 2, which is now subdivided into a large number of smaller droplets with total area $A_{2}$ (such that $A_{2} \gg A_{1}$ ), as shown in Figure 1.3. The interfacial tension $\gamma_{12}$ is the same for the large and smaller droplets as the latter are generally in the region of $0.1 \mu \mathrm{m}$ to few microns in size.

The change in free energy in going from state I to state II is made from two contributions: a surface energy term (that is positive) that is equal to $\Delta A \gamma_{12}$ (where 

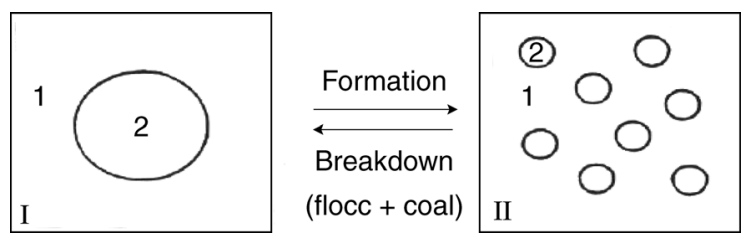

Figure 1.3 Schematic representation of emulsion formation and breakdown (see text for details).

$\Delta A=A_{2}-A_{1}$ ). An entropy of dispersions term which is also positive (since the production of a large number of droplets is accompanied by an increase in configurational entropy) which is equal to $T \Delta S^{\text {conf }}$.

From the second law of thermodynamics,

$$
\Delta G^{\text {form }}=\Delta A \gamma_{12}-T \Delta S^{\text {conf }}
$$

In most cases, $\Delta A \gamma_{12} \gg-T \Delta S^{\text {conf }}$, which means that $\Delta G^{\text {form }}$ is positive - that is, the formation of emulsions is nonspontaneous and the system is thermodynamically unstable. In the absence of any stabilization mechanism, the emulsion will break by flocculation, coalescence, Ostwald ripening, or a combination of all these processes. This situation is illustrated graphically in Figure 1.4, where several paths for emulsion breakdown processes are represented.

In the presence of a stabilizer (surfactant and/or polymer), an energy barrier is created between the droplets and therefore the reversal from state II to state I becomes noncontinuous as a result of the presence of these energy barriers. This is illustrated graphically in Figure 1.5 where, in the presence of the above energy barriers, the system becomes kinetically stable.

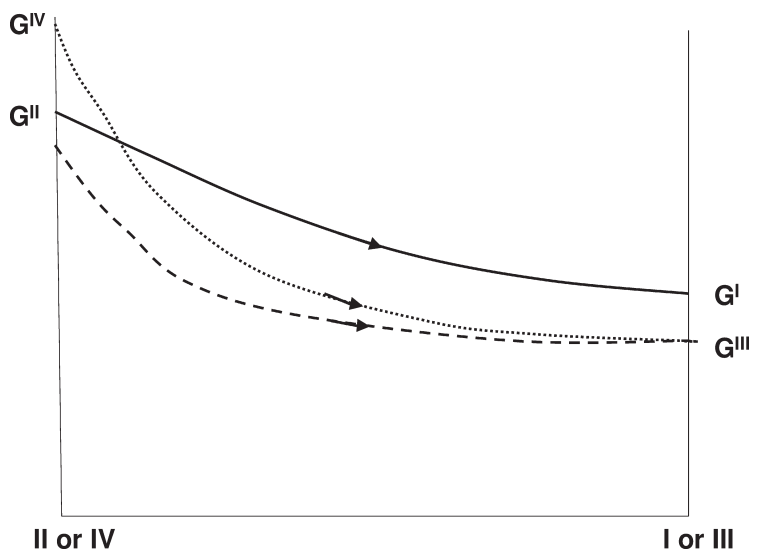

Figure 1.4 The free energy path in emulsion breakdown.

Solid line: flocculation + coalescence.

Broken line: flocculation + coalescence + sedimentation.

Dotted line: flocculation + coalescence + sedimentation + Ostwald ripening. 


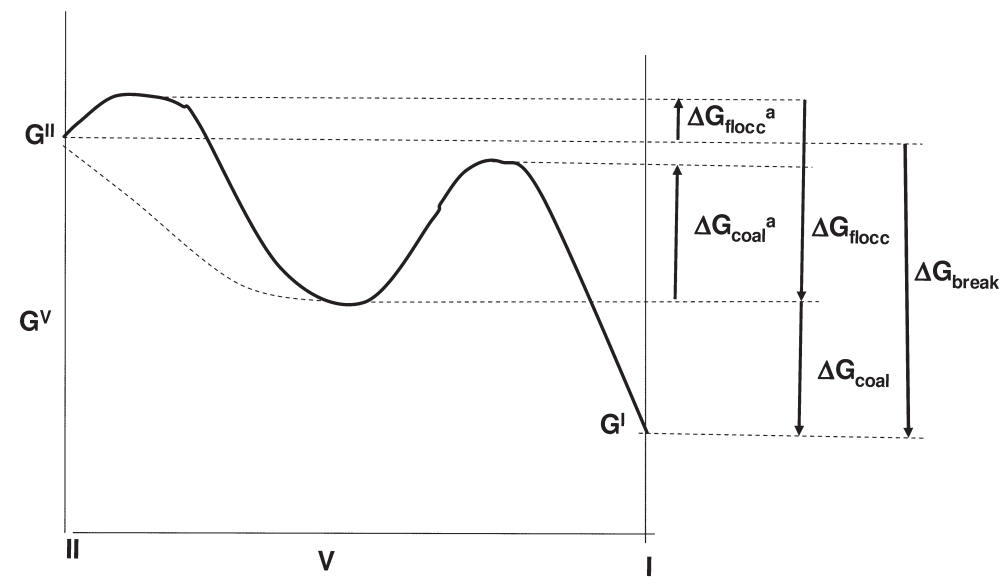

Figure 1.5 Schematic representation of the free energy path for the breakdown (flocculation and coalescence) of systems containing an energy barrier.

\section{5}

Interaction Energies (Forces) Between Emulsion Droplets and Their Combinations

Generally speaking, there are three main interaction energies (forces) between emulsion droplets, the details of which are discussed in the following sections.

\subsection{1}

\section{Van der Waals Attraction}

The van der Waals attraction between atoms or molecules are of three different types: (i) dipole-dipole (Keesom); (ii) dipole-induced dipole ((Debye-)interactions); and (iii) dispersion (London interactions). The Keesom and Debye attraction forces are vectors, and although the dipole-dipole or dipole-induced dipole attraction is large they tend to cancel due to the different orientations of the dipoles. Thus, the most important are the London dispersion interactions, which arise from charge fluctuations. With atoms or molecules consisting of a nucleus and electrons that are continuously rotating around the nucleus, a temporary dipole is created as a result of charge fluctuations. This temporary dipole induces another dipole in the adjacent atom or molecule. The interaction energy between two atoms or molecules $G_{a}$ is short range and is inversely proportional to the sixth power of the separation distance $r$ between the atoms or molecules,

$$
G_{a}=-\frac{\beta}{r^{6}}
$$

where $\beta$ is the London dispersion constant that is determined by the polarizability of the atom or molecule. 
Hamaker [4] suggested that the London dispersion interactions between atoms or molecules in macroscopic bodies (such as emulsion droplets) could be added, and this would result in a strong van der Waals attraction, particularly at close distances of separation between the droplets. For two droplets with equal radii $R$, at a separation distance $h$, the van der Waals attraction $G_{A}$ is given by the following equation (due to Hamaker),

$$
G_{A}=-\frac{A R}{12 h}
$$

where $A$ is the effective Hamaker constant,

$$
A=\left(A_{11}^{1 / 2}-A_{22}^{1 / 2}\right)^{2}
$$

and where $A_{11}$ and $A_{22}$ are the Hamaker constants of droplets and dispersion medium, respectively.

The Hamaker constant of any material depends on the number of atoms or molecules per unit volume $q$ and the London dispersion constant $\beta$,

$$
A=\pi q^{2} \beta
$$

$G_{A}$ is seen to increase very rapidly with a decrease of $h$ (at close approach). This is illustrated in Figure 1.6 which shows the van der Waals energy-distance curve for two emulsion droplets with separation distance $h$.

h

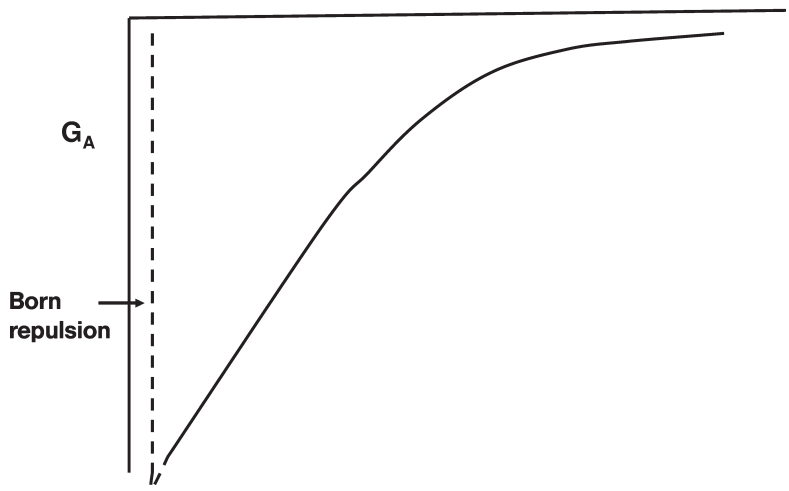

Figure 1.6 Variation of the Van der Waals attraction energy with separation distance.

In the absence of any repulsion, flocculation occurs very rapidly to produce large clusters. In order to counteract the van der Waals attraction, it is necessary to create a repulsive force. Two main types of repulsion can be distinguished depending on the nature of the emulsifier used: (i) electrostatic, which occurs due to the creation of double layers; and (ii) steric, which occurs due to the presence of adsorbed surfactant or polymer layers. 
1.5.2

Electrostatic Repulsion

This can be produced by the adsorption of an ionic surfactant, as shown in Figure 1.7, which shows a schematic representation of the structure of the double layer according to Gouy-Chapman and Stern pictures [5]. The surface potential $\psi_{\mathrm{o}}$ decreases linearly to $\psi_{\mathrm{d}}$ (Stern or zeta potential) and then exponentially with the increase of distance $x$. The double-layer extension depends on electrolyte concentration and valency (the lower the electrolyte concentration and the lower the valency, the more extended is the double layer).

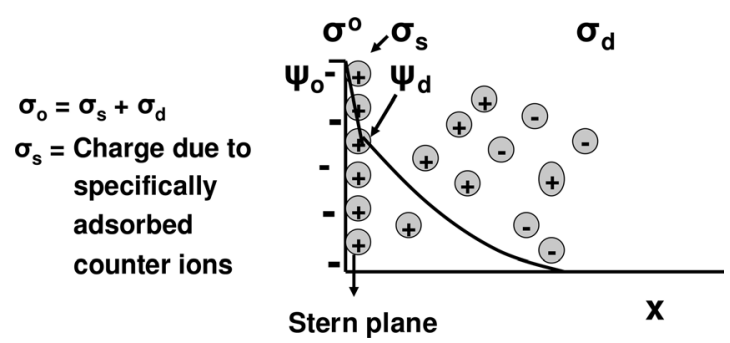

Figure 1.7 Schematic representation of double layers produced by the adsorption of an ionic surfactant.

When charged colloidal particles in a dispersion approach each other such that the double layer begins to overlap (i.e. the particle separation becomes less than twice the double-layer extension), then repulsion will occur. The individual double layers can no longer develop unrestrictedly, as the limited space does not allow complete potential decay [5, 6]. This is shown schematically in Figure 1.8 for two flat plates. This shows clearly that, when the separation distance $h$ between the emulsion droplets become smaller than twice the double-layer extension, the potential at the mid plane between the surfaces is not equal to zero (which would be the case when $h$ is more than twice the double-layer extension) plates.

The repulsive interaction $G_{e l}$ is given by the following expression:

$$
G_{e l}=2 \pi R \varepsilon_{r} \varepsilon_{o} \psi_{o}^{2} \ln [1+\exp (-\kappa h)]
$$

where $\varepsilon_{r}$ is the relative permittivity, $\varepsilon_{o}$ is the permittivity of free space, $\kappa$ is the Debye-Huckel parameter; $1 / \kappa$ is the extension of the double layer (double-layer

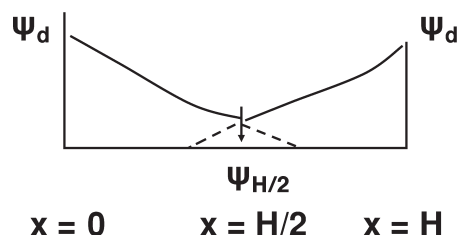

Figure 1.8 Schematic representation of a double-layer overlap. 
$10 \mid 1$ Emulsion Science and Technology: A General Introduction

thickness) that is given by the expression,

$$
\left(\frac{1}{\kappa}\right)=\left(\frac{\varepsilon_{r} \varepsilon_{o} k T}{2 n_{o} Z_{i}^{2} e^{2}}\right)
$$

where $k$ is the Boltzmann constant, $T$ is the absolute temperature, $n_{\mathrm{o}}$ is the number of ions per unit volume of each type present in bulk solution, $Z_{i}$ is the valency of the ions and $e$ is the electronic charge.

Values of $(1 / \kappa)$ at various $1: 1$ electrolyte concentrations $(C)$ are as follows:

\begin{tabular}{llllll}
\hline$C\left(\mathrm{~mol} \mathrm{dm}^{-3}\right)$ & $10^{-5}$ & $10^{-4}$ & $10^{-3}$ & $10^{-2}$ & $10^{-1}$ \\
$1 / \kappa(\mathrm{nm})$ & 100 & 33 & 10 & 3.3 & 1 \\
\hline
\end{tabular}

The double layer extension decreases with increase of electrolyte concentration, which means that the repulsion decreases with increase of electrolyte concentration, as illustrated in Figure 1.9.

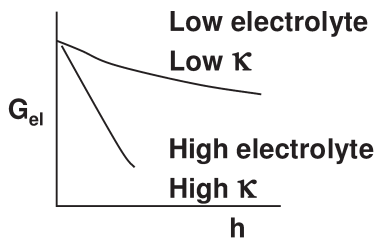

Figure 1.9 Variation of $G_{e l}$ with $h$ at low and high electrolyte concentrations ( $\left.\kappa\right)$.

A combination of van der Waals attraction and double-layer repulsion results in the well-known theory of colloid stability due to Deryaguin, Landau, Verwey and Overbeek (DLVO theory) [5, 6].

$$
G_{T}=G_{e l}+G_{A}
$$

A schematic representation of the force (energy) distance curve according to the DLVO theory is given in Figure 1.10.

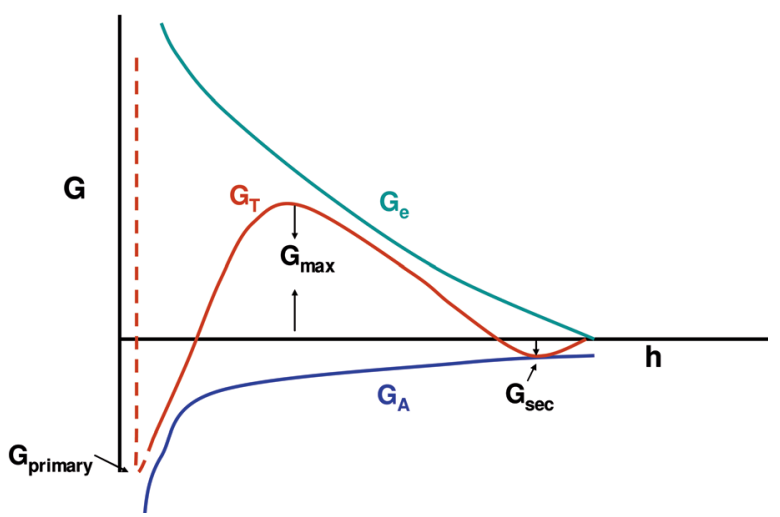

Figure 1.10 Total energy-distance curve according to the DLVO theory. 
The above presentation is for a system at low electrolyte concentration. At large $h$, attraction prevails which results in a shallow minimum $\left(G_{\text {sec }}\right)$ of the order of few $k T$ units. At very short $h, V_{A} \gg G_{e l}$, resulting in a deep primary minimum (several hundred $k T$ units). At intermediate $h, G_{e l}>G_{A}$, resulting in a maximum (energy barrier) the height of which depends on $\psi_{\mathrm{o}}($ or $\zeta$ ) and electrolyte concentration and valency. The energy maximum is usually kept $>25 \mathrm{kT}$ units, and prevents not only a close approach of the droplets but also flocculation into the primary minimum. The higher the value of $\psi_{\mathrm{o}}$ and the lower the electrolyte concentration and valency, the higher the energy maximum. At intermediate electrolyte concentrations, weak flocculation into the secondary minimum may occur.

\subsection{3}

\section{Steric Repulsion}

This is produced by using nonionic surfactants or polymers, such as alcohol ethoxylates, or A-B-A block copolymers PEO-PPO-PEO (PEO = polyethylene oxide; $\mathrm{PPO}=$ polypropylene oxide), as illustrated in Figure 1.11.
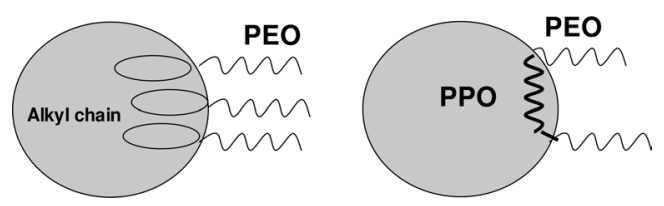

Figure 1.11 Schematic representation of the adsorbed layers.

The 'thick' hydrophilic chains (PEO in water) produce repulsion as a result of two main effects [7]:

- Unfavorable mixing of the PEO chains

When this occurs in good solvent conditions (moderate electrolyte and low temperatures) it is referred to as the osmotic or mixing free energy of interaction that is given by the expression,

$$
\frac{G_{m i x}}{k T}=\left(\frac{4 \pi}{V_{1}}\right) \phi_{2}^{2} N_{a v}\left(\frac{1}{2}-\chi\right)\left(\delta-\frac{h}{2}\right)^{2}\left(3 R+2 \delta+\frac{h}{2}\right)
$$

where $V_{1}$ is the molar volume of the solvent, $\varphi_{2}$ is the volume fraction of the polymer chain with a thickness $\delta$ and $\chi$ is the Flory-Huggins interaction parameter.

When $\chi<0.5, G_{\text {mix }}$ is positive and the interaction is repulsive; when $\chi>0.5, G_{\text {mix }}$ is negative and the interaction is attractive; when $\chi=0.5, G_{\text {mix }}=0$ and this is referred to as the $\theta$-condition.

- Entropic, volume restriction or elastic interaction, $G_{e l}$

This results from the loss in configurational entropy of the chains on significant overlap. Entropy loss is unfavorable and, therefore, $G_{e l}$ is always positive. 
A combination of $G_{m i x}, G_{e l}$ with $G_{A}$ gives the total energy of interaction $G_{T}$ (theory of steric stabilization),

$$
G_{T}=G_{m i x}+G_{e l}+G_{A}
$$

A schematic representation of the variation of $G_{m i x}, G_{e l}$ and $G_{A}$ with $h$ is shown in Figure 1.12.

In Figure 1.12 it is clear that there is only one minimum $\left(G_{\text {min }}\right)$, the depth of which depends on $R, \delta$ and $A$. When $h_{\mathrm{o}}<2 \delta$, a strong repulsion occurs and this increases very sharply with any further decrease in $h_{\mathrm{o}}$. At a given droplet size and Hamaker constant, the larger the adsorbed layer thickness, the smaller the depth of the minimum. If $G_{\min }$ is made sufficiently small (large $\delta$ and small $R$ ), then thermodynamic stability may be approached, and this explains the case with nanoemulsions.

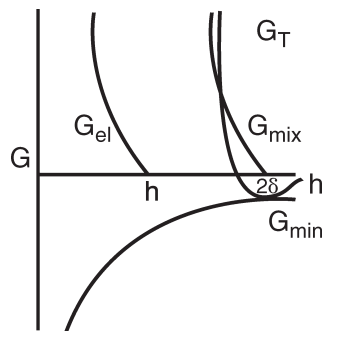

Figure 1.12 Schematic representation of the energy-distance curve for a sterically stabilized emulsion.

\section{6}

\section{Adsorption of Surfactants at the Liquid/Liquid Interface}

When surfactants accumulate at interfaces, the process is described as adsorption. The simplest interfaces are air/water $(\mathrm{A} / \mathrm{W})$ and oil/water $(\mathrm{O} / \mathrm{W})$. The surfactant molecule positions itself at the interface, with the hydrophobic portion oriented towards the hydrophobic phase (air or oil) and the hydrophilic portion oriented at the hydrophilic phase (water). This is shown schematically in Figure 1.13. As a result of adsorption, the surface tension of water is reduced from its value of $72 \mathrm{mN} \mathrm{m}^{-1}$ before adsorption to $\sim 30-40 \mathrm{mN} \mathrm{m}^{-1}$, while the interfacial tension for the $\mathrm{O} / \mathrm{W}$ system decreases from a value of $50 \mathrm{mN} \mathrm{m}^{-1}$ (for an alkane oil) before adsorption to a value of $1-10 \mathrm{mN} \mathrm{m}^{-1}$, depending on the nature of the surfactant.

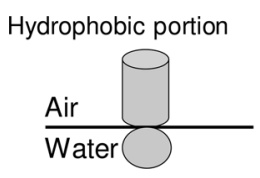

Hydrophilic portion

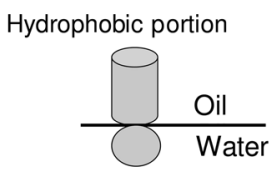

Hydrophilic portion

Figure 1.13 Schematic representation of the orientation of surfactant molecules. 
Two approaches can be applied to treat surfactant adsorption at the $\mathrm{A} / \mathrm{L}$ and $\mathrm{L} / \mathrm{L}$ interfaces [5]:

- The 'Gibbs approach', which treats the process as an equilibrium phenomenon and in which case the second law of thermodynamics can be applied.

- The 'equation of state approach', whereby the surfactant film is treated as a 'twodimensional' layer with a surface pressure $\pi$.

The Gibbs approach allows the surfactant adsorption to be obtain from surface tension measurements, whereas the equation of state approach allows the surfactant orientation to be studied the at the interface. In the following section details of only the Gibbs approach will be described.

\subsection{1}

\section{The Gibbs Adsorption Isotherm}

Gibbs derived a thermodynamic relationship between the variation of surface or interfacial tension with concentration and the amount of surfactant adsorbed $\Gamma$ (moles per unit area), referred to as the 'surface excess'. At equilibrium, the Gibbs free energy $d G^{\sigma}=0$ and the Gibbs-Deuhem equation becomes,

$$
d G^{\sigma}=-S^{\sigma} d T+A d \gamma+\sum n_{i}^{\sigma} d \mu_{i}=0
$$

At constant temperature,

$$
A d \gamma=-\sum n_{i}^{\sigma} d \mu_{i}
$$

or

$$
d \gamma=-\sum \frac{n_{i}^{\sigma}}{A} d \mu_{i}=-\sum \Gamma_{i}^{\sigma} d \mu_{i}
$$

For a surfactant (component 2) adsorbed at the surface of a solvent (component 1),

$$
-d \gamma=\Gamma_{1}^{\sigma} d \mu_{1}+\Gamma_{2}^{\sigma} d \mu_{2}
$$

If the Gibbs dividing surface is used and the assumption $\Gamma_{1}^{\sigma}=0$ is made, then

$$
-d \gamma=\Gamma_{2,1}^{\sigma} d \mu_{2}
$$

The chemical potential of the surfactant $\mu_{2}$ is given by the expression,

$$
\mu_{2}=\mu_{2}^{0}+R T \ln a_{2}^{L}
$$

where $\mu_{2}^{\circ}$ is the standard chemical potential, $a_{2}^{L}$ is the activity of surfactant that is equal to $C_{2} f_{2} \sim x_{2} f_{2}$, where $C_{2}$ is the concentration (in moles $\mathrm{dm}^{-3}$ ) and $x_{2}$ is the mole fraction that is equal to $C_{2} /\left(C_{2}+55.5\right)$ for a dilute solution and $f_{2}$ is the activity coefficient that is also $\sim 1$ in dilute solutions.

By differentiating Equation 1.20, one obtains,

$$
d \mu_{2}=R T d \ln a_{2}^{L}
$$




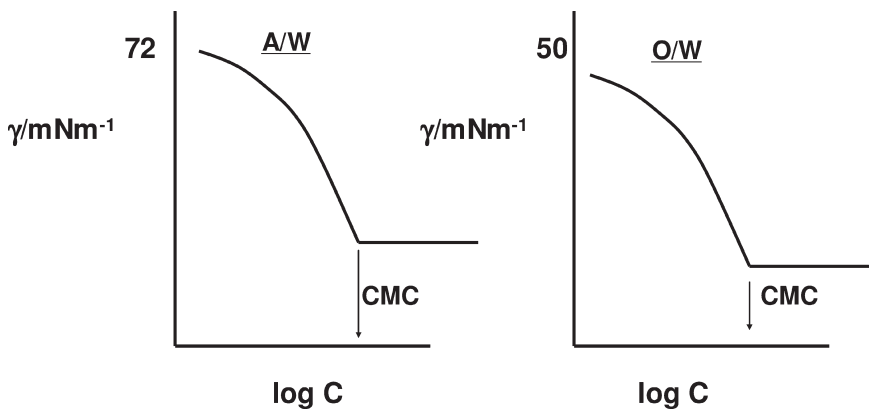

Figure 1.14 Surface or interfacial tension $(\gamma)-\log C$ curves. $C M C=$ critical micelle concentration.

Combining Equations 1.19 and 1.21,

$$
-d \gamma=\Gamma_{2,1}^{\sigma} R T d \ln a_{2}^{L}
$$

or

$$
\frac{d \gamma}{d \ln a_{2}^{L}}=-R T \Gamma_{2,1}^{L}
$$

In dilute solutions, $f_{2} \sim 1$ and,

$$
\frac{d \gamma}{d \ln C_{2}}=-\Gamma_{2} R T
$$

Equations 1.23 and 1.24 are referred to as the Gibbs adsorption equations, and show that $\Gamma_{2}$ can be determined from the experimental results of variation of $\gamma$ with $\log C_{2}$, as is illustrated in Figure 1.14 for the $\mathrm{A} / \mathrm{W}$ and $\mathrm{O} / \mathrm{W}$ interfaces.

$\Gamma_{2}$ can be calculated from the linear portion of the $\gamma-\log C$ curve just before the critical micelle concentration (CMC):

$$
\text { slope }=-\frac{d \gamma}{d \log C_{2}}=-2.303 \Gamma_{2} R T
$$

From $\Gamma_{2}$ the area per molecule of surfactant (or ion) can be calculated,

$$
\text { area } / \text { molecule }=\frac{1}{\Gamma_{2} N_{a v}}\left(\mathrm{~m}^{2}\right)=\frac{10^{18}}{\Gamma_{2} N_{a v}}\left(\mathrm{~nm}^{2}\right)
$$

where $N_{a v}$ is the Avogadro's constant (equal to $6.023 \times 10^{23}$ ).

The area per surfactant ion or molecule provides information on the orientation of the ion or molecule at the interface. The area depends on whether the molecules lie flat or vertically at the interface, and also on the length of the alkyl chain length (if the molecules lie flat) or the cross-sectional area of the head group (if the molecules lie vertically). For example, for an ionic surfactant such as sodium dodecyl sulfate (SDS), the area per molecule depends on the orientation. If the molecule lies flat, the area is determined by the area occupied by the alky chain and that by the sulfate head group. In this case the area per molecule increases with an increase in the alkyl chain length, and will be in the range $1-2 \mathrm{~nm}^{2}$. In contrast, for vertical orientation, the area per 
molecule is determined by the cross-sectional area of the sulfate group, which is $\sim 0.4 \mathrm{~nm}^{2}$, and is virtually independent of the alkyl chain length. The addition of electrolytes screens the charge on the head group, and hence the area per molecule decreases. For nonionic surfactants such as alcohol ethoxylates, the area per molecule for flat orientation is determined by the length of the alkyl chain and the number of ethylene oxide (EO) units. For vertical orientation, the area per molecule is determined by the cross-sectional area of the PEO chain, and this increases with an increase in the number of EO units.

At concentrations just before the break point, the slope of the $\gamma-\log C$ curve is constant,

$$
\left(\frac{\partial \gamma}{\partial \log C_{2}}\right)=\text { constant }
$$

which indicates that saturation of the interface occurs just below the CMC.

Above the break point $(\mathrm{C}>\mathrm{CMC})$, the slope is zero,

$$
\left(\frac{\partial \gamma}{\partial \log C_{2}}\right)=0
$$

or

$$
\gamma=\text { constant } \times \log C_{2}
$$

As $\gamma$ remains constant above the $\mathrm{CMC}$ then $C_{2}$ or $a_{2}$ of the monomer must remain constant.

The addition of surfactant molecules above the CMC must result in an association to form micelles which have low activity, and hence $a_{2}$ remains virtually constant.

The hydrophilic head group of the surfactant molecule can also affect its adsorption. These head groups can be unionized (e.g. alcohol or PEO), weakly ionized (e.g. $\mathrm{COOH}$ ), or strongly ionized (e.g. sulfates $-\mathrm{O}-\mathrm{SO}_{3}{ }^{-}$, sulfonates $-\mathrm{SO}_{3}{ }^{-}$or ammonium salts $\left.-\mathrm{N}^{+}\left(\mathrm{CH}_{3}\right)_{3}\right)$. The adsorption of the different surfactants at the $\mathrm{A} / \mathrm{W}$ and $\mathrm{O} / \mathrm{W}$ interfaces depends on the nature of the head group. With nonionic surfactants, repulsion between the head groups is smaller than with ionic head groups and adsorption occurs from dilute solutions; the CMC is low, typically $10^{-5}$ to $10^{-4} \mathrm{~mol}$ $\mathrm{dm}^{-3}$. Nonionic surfactants with medium PEO form closely packed layers at $\mathrm{C}<\mathrm{CMC}$. Adsorption is slightly affected by the moderate addition of electrolytes or a change in the $\mathrm{pH}$. Nonionic surfactant adsorption is relatively simple and can be described by the Gibbs adsorption equation.

With ionic surfactants, adsorption is more complicated, depending on the repulsion between the head groups and addition of indifferent electrolyte. The Gibbs adsorption equation must be solved to take into account the adsorption of the counterions and any indifferent electrolyte ions.

For a strong surfactant electrolyte such as $\mathrm{R}-\mathrm{O}-\mathrm{SO}_{3}{ }^{-} \mathrm{Na}^{+}\left(\mathrm{R}^{-} \mathrm{Na}^{+}\right)$:

$$
\Gamma_{2}=-\frac{1}{2 R T}\left(\frac{\partial \gamma}{\partial \ln a \pm}\right)
$$


The factor 2 in Equation 1.30 arises because both surfactant ion and counterion must be adsorbed to maintain neutrality. $(\partial \gamma / \partial \ln a \pm)$ is twice as large for an unionized surfactant molecule.

For a nonadsorbed electrolyte such as $\mathrm{NaCl}$, any increase in $\mathrm{Na}^{+} \mathrm{R}^{-}$concentration produces a negligible increase in $\mathrm{Na}^{+}$concentration $\left(\mathrm{d} \mu_{\mathrm{Na}}{ }^{+}\right.$is negligible; $\mathrm{d} \mu_{\mathrm{Cl}}{ }^{-}$is also negligible).

$$
\Gamma_{2}=-\frac{1}{R T}\left(\frac{\partial \gamma}{\partial \ln C_{N a R}}\right)
$$

which is identical to the case of nonionics.

The above analysis shows that many ionic surfactants may behave like nonionics in the presence of a large concentration of an indifferent electrolyte such as $\mathrm{NaCl}$.

\subsection{2}

\section{Mechanism of Emulsification}

As mentioned above, in order to prepare an emulsion, oil, water, a surfactant and energy are required. This can be considered from a consideration of the energy needed to expand the interface, $\Delta A \gamma$ (where $\Delta A$ is the increase in interfacial area when the bulk oil with area $A_{1}$ produces a large number of droplets with area $A_{2}$; $A_{2} \gg A_{1}, \gamma$ is the interfacial tension). Since $\gamma$ is positive, the energy needed to expand the interface is large and positive; this energy term cannot be compensated by the small entropy of dispersion $T \Delta S$ (which is also positive) and the total free energy of formation of an emulsion, $\Delta G$ given by Equation 1.5 is positive. Thus, emulsion formation is nonspontaneous and energy is required to produce the droplets.

The formation of large droplets (a few $\mu \mathrm{m}$ ), as is the case for macroemulsions, is fairly easy and hence high-speed stirrers such as the Ultra-Turrax or Silverson Mixer are sufficient to produce the emulsion. In contrast, the formation of small drops (submicron, as is the case with nanoemulsions) is difficult and requires a large amount of surfactant and/or energy. The high energy required for the formation of nanoemulsions can be understood from a consideration of the Laplace pressure $\Delta p$ (the difference in pressure between inside and outside the droplet), as given by Equations 1.3 and 1.4.

In order for a drop to be broken up into smaller droplets it must be strongly deformed, and this deformation increases $\Delta p$. This is illustrated in Figure 1.15, which shows the situation when a spherical drop deforms into a prolate ellipsoid [8].

Near point 1 there is only one radius of curvature $R_{\mathrm{a}}$, whereas near point 2 there are two radii of curvature $R_{\mathrm{b}, 1}$ and $R_{\mathrm{b}, 2}$. Consequently, the stress needed to deform the drop is higher for a smaller drop. Since the stress is generally transmitted by the surrounding liquid via agitation, higher stresses require a more vigorous agitation, and hence more energy is needed to produce smaller drops.

Surfactants play major roles in the formation of emulsions. By lowering the interfacial tension, $p$ is reduced and hence the stress required to break up a drop is reduced. Surfactants also prevent the coalescence of newly formed drops. 


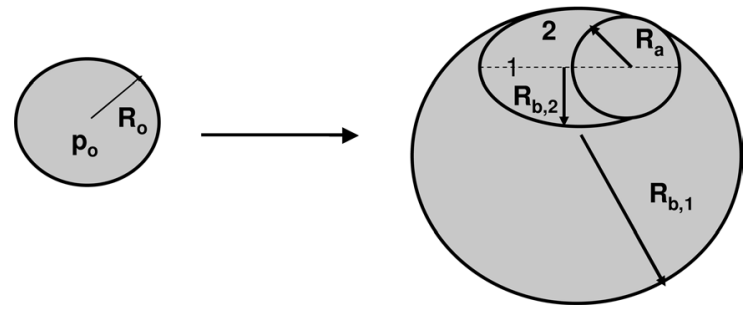

Figure 1.15 Schematic representation of the increase in Laplace pressure when a spherical drop is deformed to a prolate ellipsoid.

Figure 1.16 shows the various processes which occur during emulsification, namely the break up of the droplets, adsorption of the surfactants and droplet collision (which may or may not lead to coalescence) [8].

Each of the represented processes occurs numerous times during emulsification, and the time scale of each process is very short, typically one microsecond. This shows that the emulsification process is a dynamic process and events that occur within a microsecond range may be very important.

In order to describe emulsion formation, two main factors must be considered, namely hydrodynamics and interfacial science. To assess emulsion formation, the usual approach is to measure the droplet size distribution, using for example laser diffraction techniques. A useful average diameter $d$ is,

$$
d_{n m}=\left(\frac{S_{m}}{S_{n}}\right)^{1 /(n-m)}
$$

In most cases, $d_{32}$ (the volume/surface average or Sauter mean) is used. The width of the size distribution can be given as the variation coefficient $c_{\mathrm{m}}$, which is the

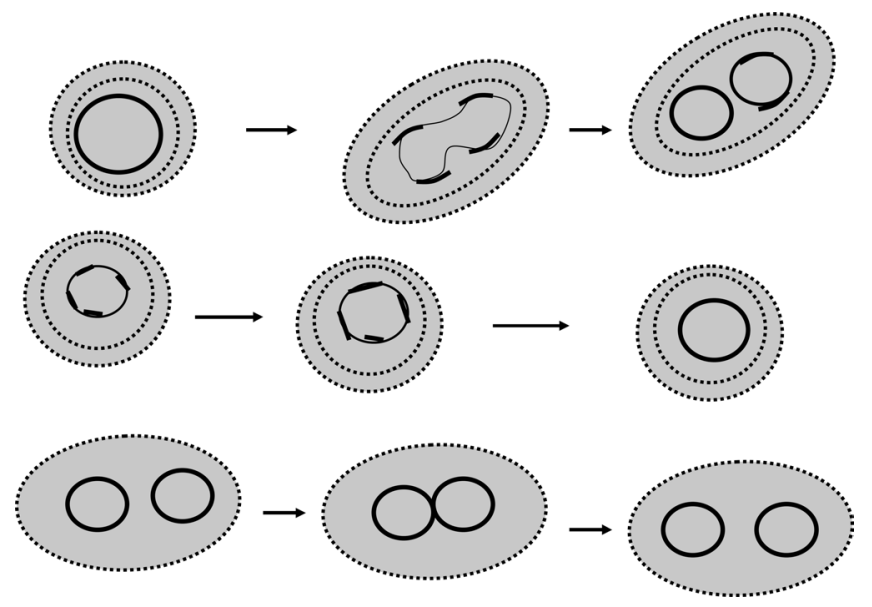

Figure 1.16 Schematic representation of the various processes occurring during emulsion formation. The drops are depicted by thin lines, and the surfactant by heavy lines and dots. 
standard deviation of the distribution weighted with $d^{\mathrm{m}}$ divided by the corresponding average $d$. Generally $C_{2}$ will be used which corresponds to $d_{32}$.

An alternative way to describe the emulsion quality is to use the specific surface area $A$ (the surface area of all emulsion droplets per unit volume of emulsion),

$$
A=\pi s^{2}=\frac{6 \phi}{d_{32}}
$$

\section{6 .3}

\section{Methods of Emulsification}

Several procedures may be applied for emulsion preparation, ranging from simple pipe flow (low agitation energy, L), static mixers and general stirrers (low to medium energy, L-M), high-speed mixers such as the Ultra-Turrax (M), colloid mills and highpressure homogenizers (high energy, $\mathrm{H}$ ), and ultrasound generators $(\mathrm{M}-\mathrm{H})$.

The method of preparation can be either continuous (C) or batch-wise (B): Pipe flow and static mixers - C; stirrers and Ultra-Turrax - B and C; colloid mill and highpressure homogenizers - C; Ultrasound - B and C.

In all methods, there is liquid flow and unbounded and strongly confined flow. In unbounded flow, any droplet is surrounded by a large amount of the flowing liquid (the confining walls of the apparatus are far away from most droplets). Thus, the forces can be either frictional (mostly viscous) or inertial. Viscous forces cause shear stresses to act on the interface between the droplets and the continuous phase (primarily in the direction of the interface). The shear stresses can be generated by either laminar flow (LV) or turbulent flow (TV), and this depends on the Reynolds number, $R_{e}$ :

$$
R_{e}=\frac{v / \rho}{\eta}
$$

where $v$ is the linear liquid velocity, $\rho$ is the liquid density and $\eta$ is its viscosity. The characteristic length $l$ is given by the diameter of flow through a cylindrical tube, and by twice the slit width in a narrow slit.

For laminar flow, $R_{e}<\sim 1000$, whereas for turbulent flow $R_{e}>\sim 2000$. Thus, whether the regime is linear or turbulent depends on the scale of the apparatus, the flow rate, and the liquid viscosity [8-11].

If the turbulent eddies are much larger than the droplets they exert shear stresses on the droplets. However, if the turbulent eddies are much smaller than the droplets then the inertial forces will cause disruption (TI).

In bounded flow, other relationships hold. For example, if the smallest dimension of that part of the apparatus where the droplets are disrupted (e.g. a slit) is comparable to the droplet size, then other relationships hold (the flow is always laminar). However, a different regime prevails if the droplets are directly injected through a narrow capillary into the continuous phase (injection regime), and membrane emulsification will occur. 
Within each regime, one essential variable is the intensity of the forces acting; thus, the viscous stress during laminar flow $\sigma_{\text {viscous }}$ is given by:

$$
\sigma_{\text {viscous }}=\eta G
$$

where $G$ is the velocity gradient.

Intensity in turbulent flow is expressed by the power density $\varepsilon$ (the amount of energy dissipated per unit volume per unit time); thus, for a laminar flow:

$$
\varepsilon=\eta G^{2}
$$

The most important regimes are: Laminar/Viscous (LV); Turbulent/Viscous (TV); and Turbulent/Inertial (TI). With water as the continuous phase, the regime is always TI, whereas for a higher viscosity of the continuous phase $\left(\eta_{C}=0.1 \mathrm{~Pa} \cdot \mathrm{s}\right)$, the regime is TV. For still higher viscosity or for a small apparatus (small l), the regime is LV, whilst for a very small apparatus (as is the case with most laboratory homogenizers) the regime is nearly always LV.

For the above regimes, a semi-quantitative theory is available that can provide the time scale and magnitude of the local stress $\sigma_{\text {ext }}$, the droplet diameter $d$, the time scale of droplet deformation $\tau_{d e f}$, the time scale of surfactant adsorption, $\tau_{a d s}$, and the mutual collision of droplets.

One important parameter that describes droplet deformation is the Weber number, $W_{e}$ (this gives the ratio of the external stress over the Laplace pressure):

$$
W_{e}=\frac{G \eta_{C} R}{2 \gamma}
$$

The viscosity of the oil plays an important role in the break-up of droplets - that is, the higher the viscosity, the greater the time taken to deform a drop. The deformation time $\tau_{d e f}$ is given by the ratio of oil viscosity to the external stress acting on the drop:

$$
\tau_{d e f}=\frac{\eta_{D}}{\sigma_{e x t}}
$$

The viscosity of the continuous phase $\eta_{C}$ plays an important role in some regimes. For a turbulent inertial regime, $\eta_{C}$ has no effect on droplet size, whereas for a turbulent viscous regime a larger $\eta_{C}$ leads to smaller droplets. For laminar viscous the effect is even stronger.

\subsection{4}

\section{Role of Surfactants in Emulsion Formation}

Surfactants lower the interfacial tension $\gamma$, which in turn causes a reduction in droplet size (the latter decreases with a decrease in $\gamma$ ). For laminar flow the droplet diameter is proportional to $\gamma$, whereas for a turbulent inertial regime the droplet diameter is proportional to $\gamma^{3 / 5}$.

The effect of reducing $\gamma$ on the droplet size is shown in Figure 1.17, where a droplet surface area $A$ and mean drop size $\mathrm{d}_{32}$ are plotted as a function of surfactant concentration $\mathrm{m}$ for various systems. 


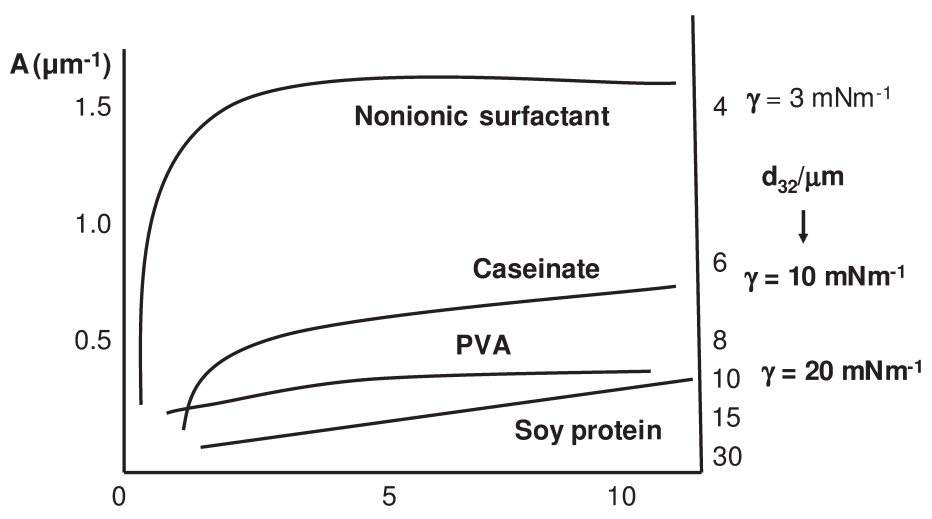

Figure 1.17 Variation of $A$ and $d_{32}$ with $m$ for various surfactant systems.

The amount of surfactant required to produce the smallest drop size will depend on its activity a (concentration) in the bulk, which in turn determines the reduction in $\gamma$, as given by the Gibbs adsorption equation:

$$
-d \gamma=R T \Gamma d \ln a
$$

where $R$ is the gas constant, $T$ is the absolute temperature and $\Gamma$ is the surface excess (the number of moles adsorbed per unit area of the interface).

$\Gamma$ increases with an increase in surfactant concentration until it eventually reaches a plateau value (saturation adsorption). This is illustrated in Figure 1.18 for various emulsifiers.

The value of $\gamma$ obtained depends on the nature of the oil and surfactant used; typically, small molecules such as nonionic surfactants reduce $\gamma$ to a greater degree than do polymeric surfactants such as polyvinyl alcohol (PVA).

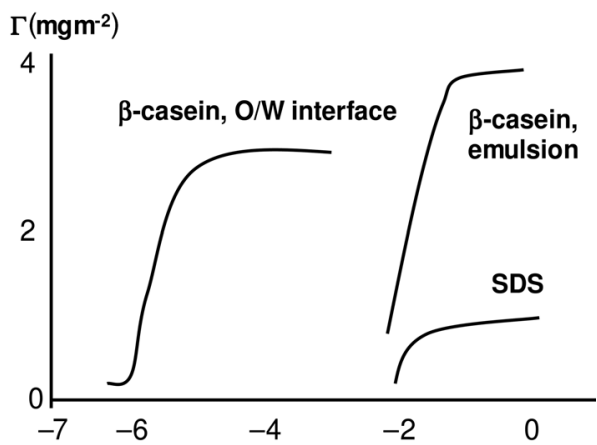

Figure 1.18 Variation of $\Gamma\left(\mathrm{mg} \mathrm{m}^{-2}\right)$ with $\log C_{\mathrm{eq}}(\mathrm{wt} \%)$. The oils are $\beta$-casein (O/W interface) toluene, $\beta$-casein (emulsions) soybean, and SDS benzene. 
Another important role of the surfactant is its effect on the interfacial dilational modulus $\varepsilon$,

$$
\varepsilon=\frac{d \gamma}{d \ln A}
$$

During emulsification, an increase in the interfacial area A occurs which in turn causes a reduction in $\Gamma$. The equilibrium is restored by the adsorption of surfactant from the bulk, but this takes time (the time is shorter at a higher surfactant activity). Thus, $\varepsilon$ is small at small at small activity and also at large activity. Because of the lack of equilibrium (or the slowness of it being achieved) with polymeric surfactants, $\varepsilon$ will not be the same for the expansion and compression of the interface.

In practice, surfactant mixtures are used which have pronounced effects on $\gamma$ and $\varepsilon$. Some specific surfactant mixtures provide lower $\gamma$ values than either of the two individual components, and the presence of more than one surfactant molecule at the interface tends to increase $\varepsilon$ at high surfactant concentrations. The various components vary in surface activity; for example, those with the lowest $\gamma$ tend to predominate at the interface, although if they are present at low concentrations it may take a long time before the lowest value is reached. Polymer-surfactant mixtures may in fact demonstrate some synergetic surface activity.

\subsection{5}

\section{Role of Surfactants in Droplet Deformation}

Apart from their effect on reducing $\gamma$, surfactants play major roles in the deformation and break up of droplets, and this may be summarized as follows. Surfactants allow the existence of interfacial tension gradients which are crucial for the formation of stable droplets. In the absence of surfactants (clean interface), the interface cannot withstand any tangential stress and the liquid motion will be continuous (Figure 1.19a).

If a liquid flows along the interface with surfactants, the latter will be swept downstream causing an interfacial tension gradient (Figure 1.19b). Hence, a balance of forces will be established:

$$
\eta\left[\frac{d V_{x}}{d y}\right]_{y=0}=-\frac{d y}{d x}
$$

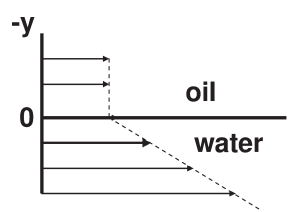

(a)

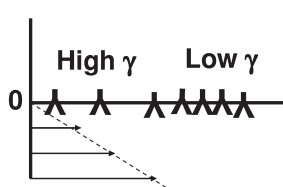

(b)

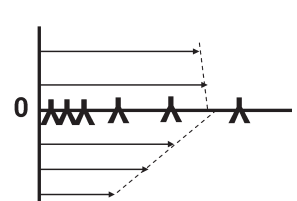

(c)

Figure 1.19 Interfacial tension gradients and flow near an oil/ water interface. (a) No surfactant; (b) the velocity gradient causes an interfacial tension gradient; (c) the interfacial tension gradient causes flow (Marangoni effect). 
$22 \mid 1$ Emulsion Science and Technology: A General Introduction

If the $\gamma$-gradient become sufficiently large it will arrest the interface. However, if the surfactant is applied at one site of the interface, a $\gamma$-gradient is formed that will cause the interface to move roughly at a velocity given by

$$
v=1.2[\eta \rho z]^{-1 / 3}|\Delta \gamma|^{2 / 3}
$$

The interface will then drag some of the bordering liquid with it (Figure 1.19c).

Interfacial tension gradients are very important in stabilizing the thin liquid film between the droplets - a step which is very important at the start of the emulsification (films of the continuous phase may be drawn through the disperse phase and the collision is very large). The magnitude of the $\gamma$-gradients and of the Marangoni effect depend on the surface dilational modulus $\varepsilon$ which, for a plane interface with one surfactant-containing phase, is given by the expression

$$
\begin{aligned}
& \varepsilon=\frac{-d \gamma / d \ln \Gamma}{\left(1+2 \xi+2 \xi^{2}\right)^{1 / 2}} \\
& \xi=\frac{d m_{C}}{d \Gamma}\left(\frac{D}{2 \omega}\right)^{1 / 2} \\
& \omega=\frac{d \ln A}{d t}
\end{aligned}
$$

where $D$ is the diffusion coefficient of the surfactant and $\omega$ represents a time scale (the time needed to double the surface area) that is approximately equal to $\tau_{d e f}$.

During emulsification, $\varepsilon$ is dominated by the magnitude of the denominator in Equation 1.43 because $\zeta$ remains small. The value of $d m_{C} / d \Gamma$ tends to go to be very high when $\Gamma$ reaches its plateau value; $\varepsilon$ goes to a maximum when $m_{C}$ is increased.

For conditions that prevail during emulsification, $\varepsilon$ increases with $m_{C}$ and is given by the relationship

$$
\varepsilon=\frac{d \pi}{d \ln \Gamma}
$$

where $\pi$ is the surface pressure $\left(\pi=\gamma_{o}-\gamma\right)$. Figure 1.20 shows the variation of $\pi$ with $\ln \Gamma$, where $\varepsilon$ is given by the slope of the line.

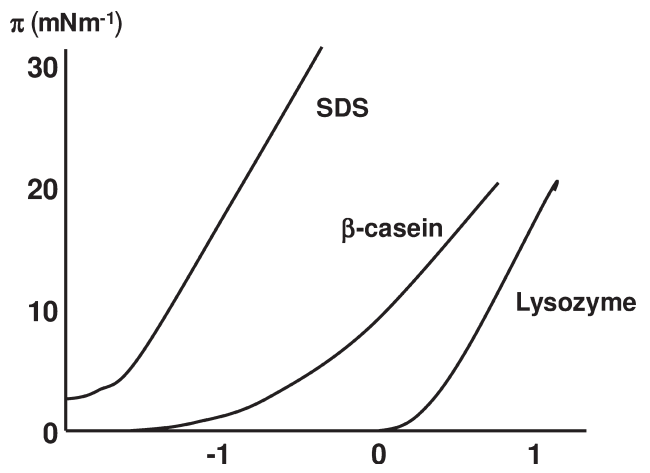

Figure 1.20 Values of $\pi$ plotted against $\ln \Gamma$ for various emulsifiers. 
Sodium dodecyl sulfate (SDS) shows a much higher $\varepsilon$-value when compared with $\beta$-casein and lysozyme; this is because the value of $\Gamma$ is higher for SDS. The two proteins show differences in their $\varepsilon$-values which may be attributed to the conformational change that occur upon adsorption.

The presence of a surfactant means that, during emulsification, the interfacial tension need not be the same everywhere (see Figure 1.19). This has two consequences: (i) the equilibrium shape of the drop is affected; and (ii) any $\gamma$-gradient formed will slow down the motion of the liquid inside the drop (this diminishes the amount of energy needed to deform and break up the drop).

Another important role of the emulsifier is to prevent coalescence during emulsification. This is clearly not due to the strong repulsion between the droplets, since the pressure at which the two drops are pressed together is much greater than the repulsive stresses. Hence, the counteracting stress must be due to the formation of $\gamma$-gradients. When two drops are pushed together, liquid will flow out from the thin layer between them; such flow will induce a $\gamma$-gradient (see Figure 1.19c), producing a counteracting stress given by

$$
\tau_{\Delta \gamma} \approx \frac{2|\Delta \gamma|}{(1 / 2) d}
$$

Here, the factor of 2 results from there being two interfaces involved. Taking a value of $\Delta \gamma=10 \mathrm{mN} \mathrm{m}^{-1}$, the stress amounts to $40 \mathrm{kPa}$ (which is of the same order of magnitude as the external stress).

Closely related to the above mechanism is the Gibbs-Marangoni effect [13-17], which is represented schematically in Figure 1.21. The depletion of surfactant in the thin film between approaching drops results in a $\gamma$-gradient without liquid flow being involved, and in turn an inward flow of liquid that tends to drive the drops apart.

The Gibbs-Marangoni effect also explains the Bancroft rule, which states that the phase in which the surfactant is most soluble forms the continuous phase. If the surfactant is in the droplets, a $\gamma$-gradient cannot develop and the drops would be prone to coalescence. Thus, surfactants with a hydrophilic-lipophilic balance (HLB) number $>7$ tend to form $\mathrm{O} / \mathrm{W}$ emulsions, while those with $\mathrm{HLB}<7$ tend to form W/O emulsions.

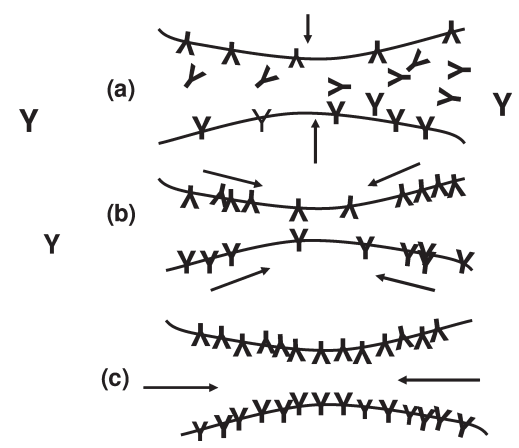

Figure 1.21 Schematic representation of the Gibbs-Marangoni effect for two approaching drops. 
The Gibbs-Marangoni effect also explains the difference between surfactants and polymers for emulsification, namely that polymers provide larger drops compared to surfactants. Polymers provide a smaller $\varepsilon$-value at low concentrations when compared to surfactants (see Figure 1.20).

Another factor which should also be considered in relation to emulsification is that of the disperse phase volume fraction, $\phi$. An increase in $\phi$ leads to increase in droplet collision and hence coalescence during emulsification. With an increase in $\phi$, the viscosity of the emulsion increases and may change the flow from turbulent to laminar (LV regime).

The presence of many particles results in a local increase in velocity gradient, which in turn means that $G$ increases. In turbulent flow, an increase in $\phi$ will induce turbulence depression and this will result in larger droplets. Turbulence depression by the addition of polymers tends to remove the small eddies, and this results in the formation of larger droplets.

If the mass ratio of surfactant to continuous phase is kept constant, an increase in $\phi$ results in a decrease in surfactant concentration and hence an increase in $\gamma_{\text {eq }}$; the result is the formation of larger droplets. However, if the mass ratio of the surfactant to disperse phase is held constant, then the above changes are reversed.

At this point it is impossible to draw any general conclusions as several of the above-mentioned mechanisms may come into play. Experiments using a highpressure homogenizer at various $\varphi$-values at constant initial $m_{C}$ (with the regime of TI changing to TV at higher $\phi$-values) showed that, with increasing $\phi(>0.1)$ the resultant droplet diameter increased and the dependence on energy consumption became weaker. A comparison of the average droplet diameter versus power consumption using different emulsifying machines, is shown in Figure 1.22. Here, it can be seen that the smallest droplet diameters were obtained when using the highpressure homogenizers.

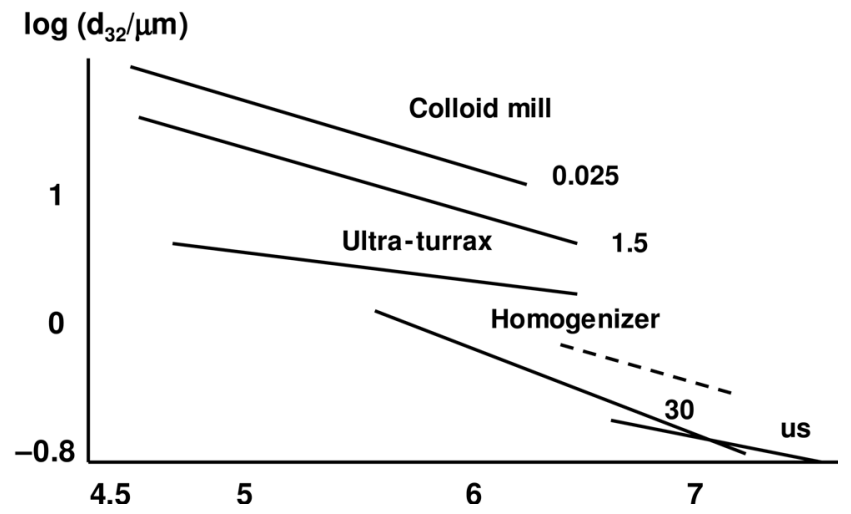

Figure 1.22 Average droplet diameters obtained in various emulsifying machines as a function of energy consumption. Numbers adjacent to the curves denote the viscosity ratio, $\lambda$. The solid lines indicate $\phi=0.04$; the broken line indicates $\phi=0.3$. us $=$ ultrasonic generator. 


\section{7}

\section{Selection of Emulsifiers}

\subsection{1}

\section{The Hydrophilic-Lipophilic Balance (HLB) Concept}

Today, the selection of different surfactants in the preparation of either $\mathrm{O} / \mathrm{W}$ or W/O emulsions is often made on an empirical basis. One such semi-empirical scale for selecting surfactants is the hydrophilic-lipophilic balance (HLB) number developed by Griffin [18]. This scale is based on the relative percentage of hydrophilic to lipophilic (hydrophobic) groups in the surfactant molecule(s). For an O/W emulsion droplet the hydrophobic chain resides in the oil phase, while the hydrophilic head group resides in the aqueous phase. In contrast, for a W/O emulsion droplet the hydrophilic group(s) reside in the water droplet, whereas the lipophilic groups reside in the hydrocarbon phase.

A guide to the selection of surfactants for particular applications is provided in Table 1.2. Here, the HLB number is seen to depend on the nature of the oil and, as an illustration, the required HLB numbers to emulsify various oils are listed in Table 1.3.

The relative importance of the hydrophilic and lipophilic groups was first recognized when using mixtures of surfactants containing varying proportions of low and high HLB numbers. The efficiency of any combination (as judged by phase separation) was found to pass a maximum when the blend contained a particular proportion of the surfactant with the higher HLB number. This is illustrated in Figure 1.23, which shows the variation of emulsion stability, droplet size and interfacial tension in relation to the percentage of surfactant with a high HLB number.

Table 1.2 A summary of surfactant HLB ranges and their applications.

\begin{tabular}{ll}
\hline HLB range & Application \\
\hline $3-6$ & W/O emulsifier \\
$7-9$ & Wetting agent \\
$8-18$ & O/W emulsifier \\
$13-15$ & Detergent \\
$15-18$ & Solubilizer \\
\hline
\end{tabular}

Table 1.3 HLB numbers required for the emulsification of various oils.

\begin{tabular}{lcc}
\hline Oil & W/O emulsion & O/W emulsion \\
\hline Paraffin oil & 4 & 10 \\
Beeswax & 5 & 9 \\
Linolin, anhydrous & 8 & 12 \\
Cyclohexane & - & 15 \\
Toluene & - & 15 \\
\hline
\end{tabular}


$26 \mid 1$ Emulsion Science and Technology: A General Introduction

The average HLB number may be calculated from additivity:

$$
\mathrm{HLB}=x_{1} \mathrm{HLB}_{1}+x_{2} \mathrm{HLB}_{2}
$$

where $x_{1}$ and $x_{2}$ are the weight fractions of the two surfactants with $\mathrm{HLB}_{1}$ and $\mathrm{HLB}_{2}$, respectively.

Griffin developed simple equations for calculating the HLB number of relatively simple nonionic surfactants. For example, in the case of a polyhydroxy fatty acid ester

$$
\mathrm{HLB}=20\left(1-\frac{S}{A}\right)
$$

where $S$ is the saponification number of the ester and $A$ is the acid number. For a glyceryl monostearate, $S=161$ and $A=198$, and the HLB is 3.8 (suitable for W/O emulsion).

For a simple alcohol ethoxylate, the HLB number can be calculated from the weight percent of ethylene oxide $(E)$ and polyhydric alcohol $(P)$ :

$$
\mathrm{HLB}=\frac{E+P}{5}
$$

If the surfactant contains PEO as the only hydrophilic group, then the contribution from one $\mathrm{OH}$ group may be neglected:

$$
\mathrm{HLB}=\frac{E}{5}
$$

For a nonionic surfactant $\mathrm{C}_{12} \mathrm{H}_{25}-\mathrm{O}-\left(\mathrm{CH}_{2}-\mathrm{CH}_{2}-\mathrm{O}\right)_{6}$, the HLB is 12 (suitable for $\mathrm{O} / \mathrm{W}$ emulsion).

The above simple equations cannot be used for surfactants containing propylene oxide or butylene oxide; neither can they be applied for ionic surfactants. Davies [19, 20] devised a method for calculating the HLB number for surfactants from their chemical formulae, using empirically determined group numbers. A group number is assigned to various component groups (a summary of the group numbers for some surfactants is shown in Table 1.4).

The HLB is then given by the following empirical equation:

$$
\text { HLB }=7+\Sigma(\text { hydrophilic group Nos })-\Sigma(\text { lipophilic group Nos })
$$

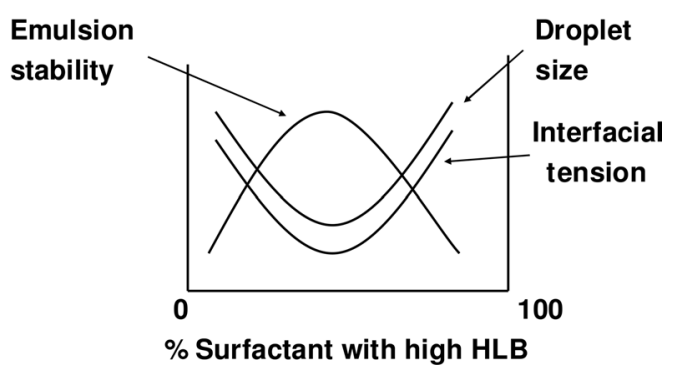

Figure 1.23 Variation of emulsion stability, droplet size and interfacial tension in relation to the percentage of surfactant with a high hydrophilic-lipophilic balance (HLB) number. 
Table 1.4 HLB group numbers.

\begin{tabular}{lc}
\hline Surfactant type/group & Group number \\
\hline Hydrophilic & 38.7 \\
$-\mathrm{SO}_{4} \mathrm{Na}^{+}$ & 21.2 \\
$-\mathrm{COO}^{-}$ & 19.1 \\
$-\mathrm{COONa}$ & 9.4 \\
$\mathrm{~N}$ (tertiary amine) & 6.8 \\
Ester (sorbitan ring) & 1.3 \\
$-\mathrm{O}-$ & 0.5 \\
$\mathrm{CH}-($ sorbitan ring) & \\
& \\
Lipophilic & 0.475 \\
(- $\mathrm{CH}_{-}$), (- $\mathrm{CH}_{2}-$ ), $\mathrm{CH}_{3}$ & \\
Derived & \\
$-\mathrm{CH}_{2}-\mathrm{CH}_{2}-\mathrm{O}$ & 0.33 \\
$-\mathrm{CH}_{2}-\mathrm{CH}_{2}-\mathrm{CH}_{2}-\mathrm{O}-$ & -0.15 \\
\hline
\end{tabular}

Davies has shown that the agreement between HLB numbers calculated from the above-described equation and those determined experimentally is quite satisfactory.

Various other procedures have been developed to obtain a rough estimate of the HLB number. Griffin found a good correlation between the cloud points of $5 \%$ solutions of various ethoxylated surfactants and their HLB numbers.

Davies [19, 20] also attempted to relate the HLB values to the selective coalescence rates of emulsions. These correlations were not realized as the emulsion stability and even its type were found to depend to a large extent on the method of dispersing the oil into the water, and vice versa. At best, the HLB number can only be used as a guide for selecting the optimum compositions of emulsifying agents.

It is possible to take any pair of emulsifying agents, which fall at opposite ends of the HLB scale, for example Tween 80 (sorbitan mono-oleate with 20 moles EO, HLB = 15) and Span 80 (sorbitan mono-oleate, $\mathrm{HLB}=5$ ) and to use them in various proportions to cover a wide range of HLB numbers. The emulsions should be prepared in the same way, with a few percent of the emulsifying blend. The stability of the emulsions is then assessed at each HLB number, either from the rate of coalescence, or qualitatively by measuring the rate of oil separation. In this way it is possible to determine the optimum HLB number for a given oil. Having found the most effective HLB value, various other surfactant pairs are compared at this HLB value, to identify the most effective pair.

\subsection{2}

\section{The Phase Inversion Temperature (PIT) Concept}

Shinoda and coworkers [21, 22] found that many O/W emulsions, when stabilized with nonionic surfactants, undergo a process of inversion at a critical temperature (known as the PIT). The PIT can be determined by following the emulsion conduc- 
tivity (a small amount of electrolyte is added to increase the sensitivity) as a function of temperature. The conductivity of the $\mathrm{O} / \mathrm{W}$ emulsion increases with increase of temperature until the PIT is reached, above which there will be a rapid reduction in conductivity (a W/O emulsion is formed). Shinoda and coworkers found that the PIT is influenced by the HLB number of the surfactant. The size of the emulsion droplets was also found to depend on the temperature and HLB number of the emulsifiers, with the droplets being more likely to coalesce when close to the PIT. However, rapid cooling of the emulsion can be used to produce a stable system. Relatively stable $\mathrm{O} / \mathrm{W}$ emulsions were obtained when the PIT of the system was $20-65^{\circ} \mathrm{C}$ higher than the storage temperature. Emulsions prepared at a temperature just below the PIT, followed by rapid cooling, generally have smaller droplet sizes. This is more easily appreciated by considering the change in interfacial tension with temperature, as illustrated in Figure 1.24. The interfacial tension is seen to decrease as the temperature is increased, to reach a minimum close to the PIT, and then to increase again. Thus, droplets prepared close to the PIT are smaller than those prepared at lower temperatures. Such droplets are also relatively unstable towards coalescence near the PIT, although by rapid cooling of the emulsion it is possible to retain the smaller size. This procedure may also be applied in the preparation of mini (nano) emulsions.

The optimum stability of the emulsion was found to be relatively insensitive to changes in the HLB value or the PIT of the emulsifier, although instability was very sensitive to the PIT of the system. It is essential, therefore to measure the PIT of the emulsion as a whole (with all other ingredients).

At a given HLB value, the stability of an emulsion against coalescence increases markedly as the molar mass of both the hydrophilic and lipophilic components increases. The enhanced stability using high-molecular-weight surfactants (polymeric surfactants) may be more easily understood from a consideration of the steric

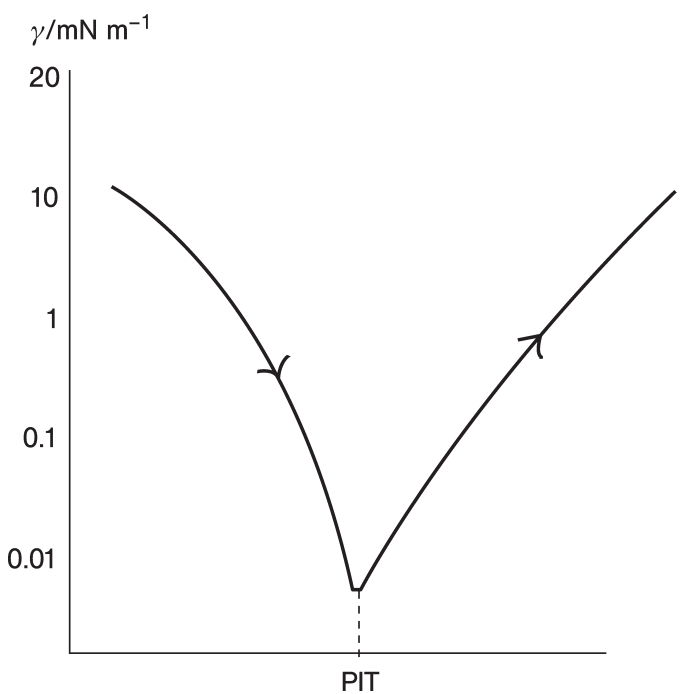

Figure 1.24 Variation of interfacial tension with temperature increase for an $\mathrm{O} / \mathrm{W}$ emulsion. 
repulsion which produces more stable films. Films produced using macromolecular surfactants resist thinning and disruption, thus reducing the possibility of coalescence. The emulsions showed maximum stability when the distribution of the PEO chains was broad; moreover, the cloud point was lower, but the PIT higher, than in the corresponding case for narrow size distributions. Thus, it is clear that the PITand HLB number are directly related parameters.

The addition of electrolytes reduces the PIT, and hence an emulsifier with a higher PIT value is required when preparing emulsions in the presence of electrolytes. Electrolytes cause dehydration of the PEO chains, and in effect this reduces the cloud point of the nonionic surfactant. It is necessary to compensate for this effect by using a surfactant with a higher HLB. The optimum PIT of the emulsifier is fixed if the storage temperature is fixed.

In view of the above correlation between PIT and HLB, and the possible dependence of the kinetics of droplet coalescence on the HLB number, Sherman and coworkers have suggested the use of PIT measurements as a rapid method for assessing emulsion stability. However, care must be taken when using such methods to assess the long-term stability as the correlations were based on a very limited number of surfactants and oils.

Measurement of the PITcan at best be used as a guide for the preparation of stable emulsions. An assessment of the stability should be made by following the droplet size distribution as a function of time using either a Coulter counter or lightdiffraction techniques. An alternative method of assessing stability against coalescence may be to follow the rheology of the emulsion as a function of time and temperature. However, care should be taken when analyzing the rheological data, as coalescence may result in an increase in droplet size which is usually followed by a reduction in the viscosity of the emulsion. This trend is only observed if the coalescence is not accompanied by flocculation of the emulsion droplets (which results in an increase in the viscosity). Ostwald ripening may also complicate the analysis of rheological data.

\subsection{3}

\section{The Cohesive Energy Ratio (CER) Concept}

Beerbower and Hill [23] considered the dispersing tendency on the oil and water interfaces of the surfactant or emulsifier in terms of the ratio of the cohesive energies of the mixtures of oil with the lipophilic portion of the surfactant and the water with the hydrophilic portion. These authors used the Winsor $R_{o}$ concept, which is the ratio of the intermolecular attraction of oil molecules (O) and the lipophilic portion of surfactant $(\mathrm{L}), C_{L O}$, to that of water $(\mathrm{W})$ and hydrophilic portion $(\mathrm{H}), C_{H W}$

$$
R_{o}=\frac{C_{L O}}{C_{H W}}
$$

Several interaction parameters may be identified at the oil and water sides of the interface. Typically, at least nine interaction parameters can be identified, as shown schematically in Figure 1.25. 


$$
\begin{aligned}
& \mathrm{C}_{\mathrm{LL}}, \mathrm{C}_{\mathrm{OO}}, \mathrm{C}_{\mathrm{LO}} \text { (at oil side) } \\
& \mathrm{C}_{\mathrm{HH}}, \mathrm{C}_{\mathrm{WW}}, \mathrm{C}_{\mathrm{HW}} \text { (at water side) } \\
& \mathrm{C}_{\mathrm{LW}}, \mathrm{C}_{\mathrm{HO}}, \mathrm{C}_{\mathrm{LH}} \text { (at oil interface) }
\end{aligned}
$$

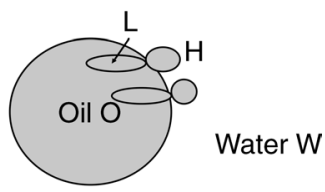

Figure 1.25 The Cohesive Energy Ratio (CER) concept.

In the absence of an emulsifier, there will be only three interaction parameters, namely $C_{O O}, C_{W W}$ and $C_{O W}$; then, if $C_{O W} \ll C_{W W}$, the emulsion will break.

The above interaction parameters may be related to the Hildebrand solubility parameter [24] $\delta$ (at the oil side of the interface) and the Hansen [25] nonpolar, hydrogen bonding and polar contributions to $\delta$ at the water side of the interface. The solubility parameter of any component is related to its heat of vaporization $\Delta H$ by the expression

$$
\delta^{2}=\frac{\Delta H-R T}{V_{m}}
$$

where $V_{m}$ is the molar volume.

Hansen considered $\delta$ (at the water side of the interface) to consist of three main contributions: a dispersion contribution, $\delta_{d}$; a polar contribution, $\delta_{p}$; and a hydrogen bonding contribution, $\delta_{h}$, each of which have different weighting factors:

$$
\delta^{2}=\delta_{d}^{2}+0.25 \delta_{p}^{2}+\delta_{h}^{2}
$$

Beerbower and Hills used the following expression for the HLB number:

$$
\mathrm{HLB}=20\left(\frac{M_{H}}{M_{L}+M_{H}}\right)=20\left(\frac{V_{H} \rho_{H}}{V_{L} \rho_{L}+V_{H} \rho_{H}}\right)
$$

where $M_{H}$ and $M_{L}$ are the molecular weights of the hydrophilic and lipophilic portions of the surfactants, respectively, $V_{L}$ and $V_{H}$ are their corresponding molar volumes, respectively, and $\rho_{H}$ and $\rho_{L}$ are the densities, respectively.

The cohesive energy ratio was originally defined by Winsor (see Equation 1.53). When $C_{L O}>C_{H W}$, then $R>1$ and a W/O emulsion forms, but if $C_{L O}<C_{H W}$, then $R<1$ and an $\mathrm{O} / \mathrm{W}$ emulsion forms. Yet, if $C_{L O}=C_{H W}$, then $R=1$ and a planar system results; this denotes the inversion point.

$R_{o}$ can be related to $V_{L}, \delta_{L}$ and $V_{H}, \delta_{H}$ by the expression

$$
R_{o}=\frac{V_{L} \delta_{L}^{2}}{V_{H} \delta_{H}^{2}}
$$

From Equation 1.57,

$$
R_{o}=\frac{V_{L}\left(\delta_{d}^{2}+0.25 \delta_{p}^{2}+0.25 \delta_{h}^{2}\right)_{L}}{V_{h}\left(\delta_{d}^{2}+0.25 \delta_{p}^{2}+0.25 \delta_{h}^{2}\right)_{H}}
$$


By combining Equations 1.57 and 1.58 one obtains the following general expression for the CER:

$$
R_{o}=\left(\frac{20}{\mathrm{HLB}}-1\right) \frac{\rho_{h}\left(\delta_{d}^{2}+0.25 \delta_{p}^{2}+0.25 \delta_{h}^{2}\right)_{L}}{\rho_{L}\left(\delta_{d}^{2}+0.25 \delta_{p}^{2}+0.25 \delta_{p}^{2}\right)_{H}}
$$

For an $\mathrm{O} / \mathrm{W}$ system, HLB $=12-15$ and $R_{o}=0.58-0.29\left(R_{o}<1\right)$, while for a W/O system HLB $=5-6$ and $R_{o}=2.3-1.9\left(R_{o}>1\right)$. For a planar system, HLB $=8-10$ and $R_{o}=1.25-0.85\left(R_{o} \sim 1\right)$.

The $R_{o}$ equation combines both the HLB and cohesive energy densities, and also provides a more quantitative estimate of emulsifier selection. Moreover, $R_{o}$ takes into consideration the HLB, molar volume and chemical match, with the success of the approach depending on the availability of data on the solubility parameters of the various surfactant portions. Some values of these parameters are provided elsewhere [26].

\subsection{4}

\section{The Critical Packing Parameter for Emulsion Selection}

The critical packing parameter (CPP) is a geometric expression which relates the hydrocarbon chain volume $(v)$ and length $(l)$, and also the interfacial area occupied by the head group (a) [27]:

$$
\mathrm{CPP}=\frac{v}{l_{c} a_{o}}
$$

where $a_{o}$ is the optimal surface are per head group and $l_{c}$ is the critical chain length.

Regardless of the shape of any aggregated structure (spherical or cylindrical micelle, or a bilayer), no point within the structure can be farther from the hydrocarbon-water surface than $l_{c}$. The critical chain length, $l_{c}$, is roughly equal but less than the fully extended length of the alkyl chain.

The above concept can be applied to predict the shape of an aggregated structure. If we consider a spherical micelle with radius $r$ and aggregation number $n$, then the volume of the micelle is given by

$$
\left(\frac{4}{3}\right) \pi r^{3}=n v
$$

where $v$ is the volume of a surfactant molecule.

The area of the micelle is then given by

$$
4 \pi r^{2}=n_{a_{o}}
$$

where $a_{o}$ is the area per surfactant head group. By combining Equations 1.61 and 1.62 we obtain

$$
a_{0}=\frac{3 v}{r}
$$


where the cross-sectional area of the hydrocarbon chain, $a$, is given by the ratio of its volume to its extended length $l_{c}$ :

$$
a=\frac{v}{l_{c}}
$$

From Equations 1.63 and 1.64,

$$
\mathrm{CPP}=\frac{a}{a_{0}}=\left(\frac{1}{3}\right)\left(\frac{r}{l_{c}}\right)
$$

Since $r<l_{c}$, then $\mathrm{CPP} \leq(1 / 3)$.

For a cylindrical micelle with length $d$ and radius $r$,

Volume of the micelle $=\pi \cdot r^{2} \cdot d=n v$

Area of the micelle $=2 \pi \cdot r \cdot d=n a_{o}$

By combining Equations 1.66 and 1.67,

$$
\begin{aligned}
& a_{o}=\frac{2 v}{r} \\
& a=\frac{v}{l_{c}} \\
& \mathrm{CPP}=\frac{a}{a_{o}}=\left(\frac{1}{2}\right)\left(\frac{r}{l_{c}}\right)
\end{aligned}
$$

Since $r<l_{c}$, then $(1 / 3)<\mathrm{CPP} \leq(1 / 2)$. For vesicles (liposomes), $1>\mathrm{CPP} \geq(2 / 3)$, while for lamellar micelles $P \sim 1$.

Surfactants that make spherical micelles with the above packing constraints [i.e. $\mathrm{CPP} \leq(1 / 3)]$ are more suitable for $\mathrm{O} / \mathrm{W}$ emulsions, whereas surfactants with $\mathrm{CPP}>1$ (i.e. forming inverted micelles) are suitable for the formation of W/O emulsions.

\section{8}

\section{Creaming or Sedimentation of Emulsions}

This is the result of gravity, when the density of the droplets and the medium are not equal. When the density of the disperse phase is less than that of the medium then creaming will occur; however, if the density of the disperse phase is greater than that of the medium then sedimentation will occur. Figure 1.24 gives A schematic representation of the creaming of emulsions for these three cases is shown in Figure 1.26 [28].

In Figure 1.26, case (a) represents the situation for small droplets $(<0.1 \mu \mathrm{m}$, i.e. nanoemulsions) whereby the Brownian diffusion $k T$ (where $k$ is the Boltzmann 


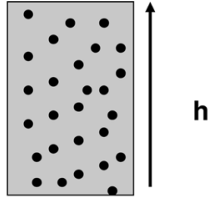

(a) $k T>(4 / 3) \pi R^{3} \Delta \rho g h$

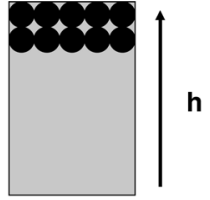

(b) $k T<(4 / 3) \pi R^{3} \Delta \rho g h$

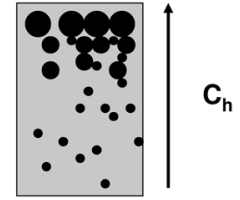

(c) $C_{h}=C_{o} \exp (-m g h / k T)$

$$
\begin{aligned}
C_{0}=\text { conc.; at } t=0 \\
C_{h}=\text { conc.; at time } t \text { and } \\
\text { height } h \\
m=(4 / 3) \pi R^{3} \Delta \rho
\end{aligned}
$$

Figure 1.26 Schematic representation of the creaming of emulsions.

constant and $T$ is the absolute temperature) exceeds the force of gravity (mass $\times$ acceleration due to gravity, g):

$$
k T \gg \frac{4}{3} \pi R^{3} \Delta \rho g L
$$

where $R$ is the droplet radius, $\Delta \rho$ is the density difference between the droplets and the medium, and $L$ is the height of the container.

Case (b) in Figure 1.26 represents emulsions consisting of 'monodisperse' droplets with radius $>1 \mu \mathrm{m}$. In this case, the emulsion separates into two distinct layers, with the droplets forming a cream or sediment and leaving the clear supernatant liquid. However, this situation is seldom observed in practice.

Case (c) in Figure 1.26 is that for a polydisperse (practical) emulsion, where the droplets will cream or sediment at various rates. In the latter case a concentration gradient build-up will occur, with the larger droplets staying either at the top of the cream layer or at the bottom of the sediment:

$$
C(h)=C_{o} \exp \left(-\frac{m g h}{k T}\right)
$$

where $C(h)$ is the concentration (or volume fraction $\phi$ ) of the droplets at height $h$, while $C_{o}$ is the concentration at zero time, which is the same at all heights.

\subsection{1}

\section{Creaming or Sedimentation Rates}

Here, three situations must be taken into account.

Very Dilute Emulsions $(\phi<\mathbf{0 . 0 1})$ In this case the rate could be calculated using Stokes' law, which balances the hydrodynamic force with gravity force

$$
\text { Hydrodynamic force }=6 \pi \eta_{0} R v_{0}
$$

$$
\text { Gravity force }=\frac{4}{3} \pi R^{3} \Delta \rho g
$$




$$
v_{o}=\frac{2}{9} \frac{\Delta \rho g R^{2}}{\eta_{o}}
$$

where $v_{o}$ is the Stokes' velocity and $\eta_{o}$ is the viscosity of the medium.

For an $\mathrm{O} / \mathrm{W}$ emulsion with $\Delta \rho=0.2$ in water $\left(\eta_{0} \sim 10^{-3} \mathrm{~Pa} \cdot \mathrm{s}\right)$, the rate of creaming or sedimentation is $\sim 4.4 \times 10^{-5} \mathrm{~ms}^{-1}$ for $10 \mu \mathrm{m}$ droplets, and $\sim 4.4 \times 10^{-7} \mathrm{~ms}^{-1}$ for $1 \mu \mathrm{m}$ droplets. This means that, in a $0.1 \mathrm{~m}$ container, creaming or sedimentation of the $10 \mu \mathrm{m}$ droplets is complete in $\sim 0.6 \mathrm{~h}$, but for the $1 \mu \mathrm{m}$ droplets this takes $\sim 60 \mathrm{~h}$.

Moderately Concentrated Emulsions $(0.2<\phi<0.1)$ In this case one must take into account the hydrodynamic interaction between the droplets, which reduces the Stokes' velocity $(v)$ to a value given by the following expression [29]:

$$
v=v_{o}(1-k \phi)
$$

where $k$ is a constant that accounts for hydrodynamic interaction, and is of the order of 6.5 ; this means that the rate of creaming or sedimentation is reduced by about $65 \%$.

Concentrated Emulsions $(\phi>0.2) \quad$ The rate of creaming or sedimentation becomes a complex function of $\phi$, as illustrated in Figure 1.27, which also shows the change of relative viscosity $\eta_{r}$ with $\phi$. As can also be seen from Figure 1.27, $v$ decreases with increase in $\phi$ and ultimately approaches zero when $\phi$ exceeds a critical value, $\varphi_{p}$, which is the so-called 'maximum packing fraction'. The value of $\phi_{p}$ for monodisperse 'hard-spheres' ranges from 0.64 (for random packing) to 0.74 for hexagonal packing, and exceeds 0.74 for polydisperse systems. Also, for emulsions which are deformable, $\phi_{p}$ can be much larger than 0.74 .

The data in Figure 1.27 also show that when $\phi$ approaches $\phi_{p}, \eta_{r}$ approaches $\infty$. In practice, most emulsions are prepared at $\phi$ values well below $\phi_{p}$, usually in the range $0.2-0.5$, and under these conditions creaming or sedimentation is the rule rather than the exception. Several procedures may be applied to reduce or eliminate creaming or sedimentation, and these are discussed below.

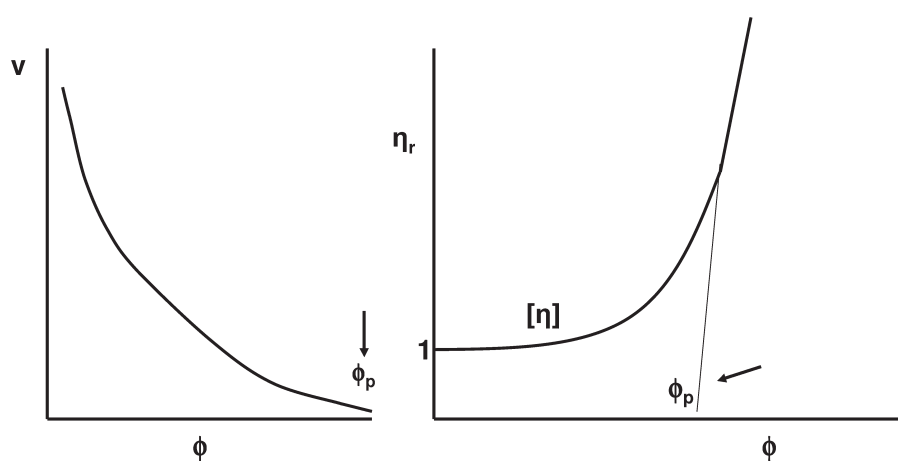

Figure 1.27 Variation of $v$ and $\eta_{r}$ with $\phi$. 


\section{8 .2}

\section{Prevention of Creaming or Sedimentation}

Matching Density of Oil and Aqueous Phases Clearly, if $\Delta \rho=0$, then $v=0$; however, this method is seldom practical as density matching (if possible) occurs at only one temperature.

Reduction of Droplet Size As the gravity force is proportional to $R^{3}$, then if $R$ is reduced by a factor of 10 the gravity force is reduced by 1000 . Below a certain droplet size (which also depends on the density difference between oil and water), the Brownian diffusion may exceed gravity, and creaming or sedimentation is prevented. This is the principle of the formulation of nanoemulsions (with size range $20-200 \mathrm{~nm}$ ) that may show very little or no creaming or sedimentation. The same applies for microemulsions (size range $5-50 \mathrm{~nm}$ ).

Use of 'Thickeners' These are high-molecular-weight polymers, and may be either natural or synthetic; examples include xanthan gum, hydroxyethyl cellulose, alginates and carragenans. In order to understand the role of these 'thickeners', we should first consider the gravitational stresses exerted during creaming or sedimentation:

$$
\text { Stress }=\text { mass of drop } \times \text { acceleration of gravity }=\frac{4}{3} \pi R^{3} \Delta \rho g
$$

In order to overcome such stress, a restoring force is needed:

$$
\text { Restoring force }=\text { area of drop } \times \text { stress of drop }=4 \pi R^{3} \sigma_{p}
$$

Thus, the stress exerted by the droplet $\sigma_{p}$ is given by

$$
\sigma_{p}=\frac{\Delta \rho \operatorname{Rg}}{3}
$$

Simple calculation shows that $\sigma_{p}$ is in the range of $10^{-3}$ to $10^{-1} \mathrm{~Pa}$, which implies that for the prediction of creaming or sedimentation there is a need to measure the viscosity at such low stresses. This can be achieved by using constant stress or creep measurements.

The above-described 'thickeners' satisfy the criteria for obtaining very high viscosities at low stresses or shear rates; this can be illustrated from plots of shear stress $\sigma$ and viscosity $\eta$ versus shear rate $\dot{\gamma}$ (or shear stress), as shown in Figure 1.28. These systems are described as either 'pseudoplastic' or 'shear thinning'. The low-shear (residual or zero shear rate) viscosity $\eta_{(\mathrm{o})}$ can reach several thousand Pas, and such high values prevent either creaming or sedimentation [30, 31].

The behavior described in Figure 1.28 is obtained above a critical polymer concentration $\left(C^{*}\right)$, which can be located from plots of $\log \eta$ versus $\log C$, as shown in Figure 1.29. 


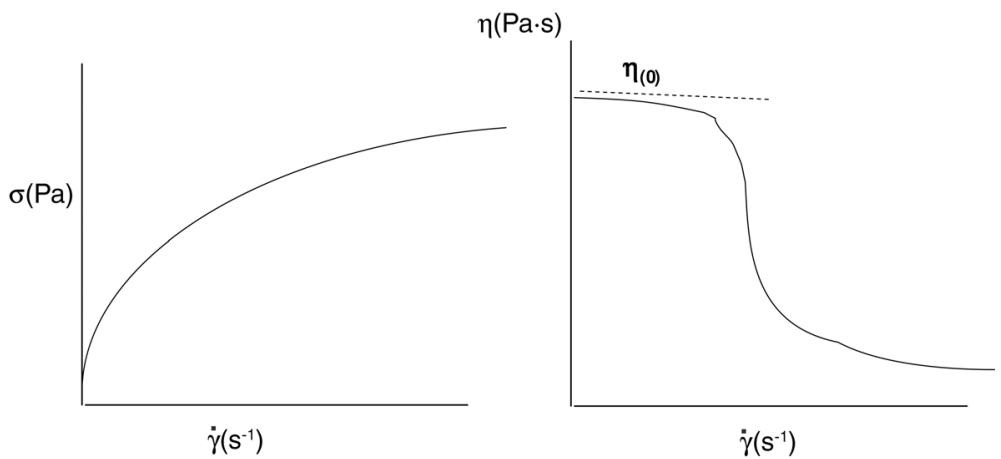

Figure 1.28 Variation of (stress) $\sigma$ and viscosity $\eta$ with shear rate $\dot{\gamma}$.

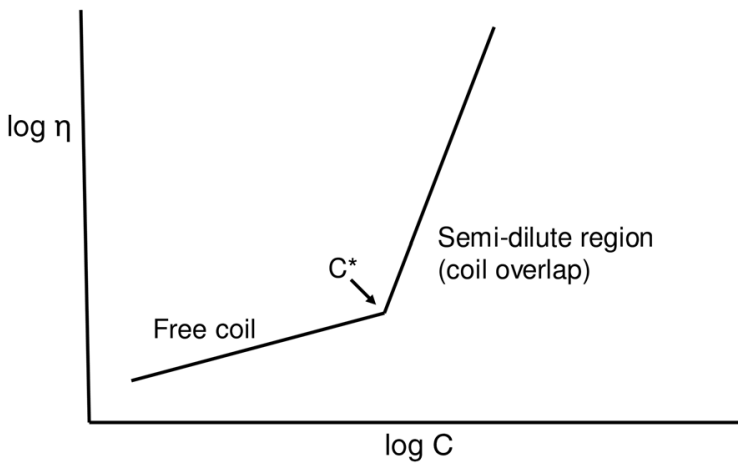

Figure 1.29 Variation of $\log \eta$ with $\log C$ for polymer solutions.

Below $C^{*}$ the $\log \eta-\log C$ curve has a slope in the region of 1 , whereas above $C^{*}$ the slope of the line exceeds 3 . In most cases a good correlation between the rate of creaming or sedimentation and $\eta_{(o)}$ is obtained.

Controlled Flocculation As discussed earlier, the total energy-distance of the separation curve for electrostatically stabilized shows a shallow minimum (secondary minimum) at relatively long distance of separation between the droplets. By adding small amounts of electrolyte, such a minimum can be made sufficiently deep for weak flocculation to occur. The same applies to sterically stabilized emulsions, which show only one minimum, the depth of which can be controlled by reducing the thickness of the adsorbed layer. This can be achieved by reducing the molecular weight of the stabilizer and/or the addition of a nonsolvent for the chains (e.g. electrolyte).

The above phenomenon of weak flocculation may be applied to reduce creaming or sedimentation, although in practice this is not easy as there is also a need to control the droplet size. 
$\propto \propto$

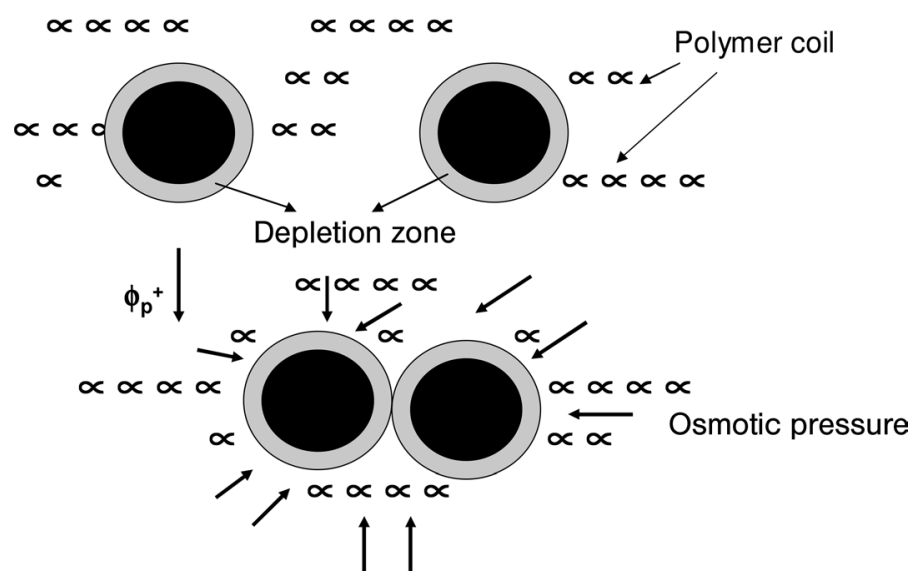

Figure 1.30 Schematic representation of depletion flocculation.

Depletion Flocculation This is achieved by the addition of 'free' (nonadsorbing) polymer in the continuous phase [32]. At a critical concentration, or volume fraction of free polymer, $\phi_{p}^{+}$, weak flocculation occurs, as the free polymer coils become 'squeezed-out' from between the droplets. This is illustrated in Figure 1.30, which shows the situation when the polymer volume fraction exceeds the critical concentration. Here, the osmotic pressure outside the droplets is higher than in between the droplets, and this results in an attraction the magnitude of which depends on the concentration of the free polymer and its molecular weight, as well as on the droplet size and $\phi$. The value of $\phi_{p}^{+}$decreases not only with an increase in the molecular weight of the free polymer, but also as the volume fraction of the emulsion increases.

The weak flocculation shown in Figure 1.30 can be applied to reduce creaming or sedimentation, although it suffers from the following drawbacks:

- Temperature dependence: as the temperature increases, the hydrodynamic radius of the free polymer decreases (due to dehydration) and hence more polymer will be required to achieve the same effect at lower temperatures.

- If the free polymer concentration is increased above a certain limit, phase separation may occur and the flocculated emulsion droplets may cream or sediment faster than in the absence of the free polymer.

\section{9}

\section{Flocculation of Emulsions}

Flocculation is the result of van der Waals attraction that is universal for all disperse systems. The van der Waals attraction $G_{A}$ was described earlier (see Figure 1.5), and is inversely proportional to the droplet-droplet distance of separation $h$; it also depends 
on the effective Hamaker constant $A$ of the emulsion system. The primary way of overcoming the van der Waals attraction is by electrostatic stabilization using ionic surfactants; this results in the formation of electrical double layers that introduce a repulsive energy which overcomes the attractive energy. Emulsions stabilized by electrostatic repulsion become flocculated at intermediate electrolyte concentrations (see below). A second - and most effective - method of overcoming flocculation is by 'steric stabilization', using nonionic surfactants or polymers. Here, stability may be maintained in electrolyte solutions (as high as $1 \mathrm{~mol} \mathrm{dm}^{-3}$, depending on the nature of the electrolyte) and up to high temperatures (in excess of $50^{\circ} \mathrm{C}$ ), provided that the stabilizing chains (e.g. PEO) are still under better than $\theta$-conditions $(\chi<0.5)$.

\subsection{1}

\section{Mechanism of Emulsion Flocculation}

This can occur if the energy barrier is small or absent (for electrostatically stabilized emulsions), or when the stabilizing chains reach poor solvency (for sterically stabilized emulsions, $\chi>0.5$ ). For convenience, the flocculation of electrostatically and sterically stabilized emulsions will be discussed separately.

\subsubsection{Flocculation of Electrostatically Stabilized Emulsions}

As discussed earlier, the condition for kinetic stability is $G_{\max }>25 k T$, but when $G_{\max }<5 k T$, then flocculation will occur. Two types of flocculation kinetics may be distinguished: (i) fast flocculation with no energy barrier; and (ii) slow flocculation, when an energy barrier exists.

Fast Flocculation Kinetics These were treated by Smoluchowski [33], who considered the process to be represented by second-order kinetics and the process to be simply diffusion-controlled. The number of particles $n$ at any time $t$ may be related to the final number (at $t=0$ ), $n_{o}$, by the following expression:

$$
n=\frac{n_{o}}{1+k n_{0} t}
$$

where $k$ is the rate constant for fast flocculation that is related to the diffusion coefficient of the particles $D$. That is:

$$
k=8 \pi D R
$$

where $D$ is given by the Stokes-Einstein equation

$$
D=\frac{k T}{6 \pi \eta R}
$$

By combining Equations 1.81 and 1.82, we get

$$
k=\frac{4}{3} \frac{k T}{\eta}=5.5 \times 10^{-18} \mathrm{~m}^{3} \mathrm{~s}^{-1} \text { for water at } 25^{\circ} \mathrm{C}
$$


Table 1.5 Half-life values of emulsion flocculation.

\begin{tabular}{lllll}
\hline & \multicolumn{3}{c}{$\phi$-Value } \\
\cline { 2 - 5 } & $10^{-5}$ & $10^{-2}$ & $\mathbf{1 0}^{-1}$ & $\mathbf{5} \times 10^{-1}$ \\
\hline 0.1 & $765 \mathrm{~s}$ & $76 \mathrm{~ms}$ & $7.6 \mathrm{~ms}$ & $1.5 \mathrm{~ms}$ \\
1.0 & $21 \mathrm{~h}$ & $76 \mathrm{~s}$ & $7.6 \mathrm{~s}$ & $1.5 \mathrm{~s}$ \\
10.0 & $4 \mathrm{months}$ & $21 \mathrm{~h}$ & $2 \mathrm{~h}$ & $25 \mathrm{~min}$ \\
\hline
\end{tabular}

The half life $t_{1 / 2}\left[n=(1 / 2) n_{o}\right]$ can be calculated at various $n_{o}$ or volume fractions $\phi$, as shown in Table 1.5 .

Slow Flocculation Kinetics These were treated by Fuchs [34], who related the rate constant $k$ to the Smoluchowski rate by the stability constant W:

$$
\mathrm{W}=\frac{k_{0}}{k}
$$

$W$ is related to $G_{\max }$ by the following expression [35]:

$$
W=\frac{1}{2} \exp \left(\frac{G_{\max }}{k T}\right)
$$

As $G_{\max }$ is determined by the salt concentration $C$ and valency, it is possible to derive an expression relating $W$ to $C$ and $Z$ :

$$
\log W=-2.06 \times 10^{9}\left(\frac{R \gamma^{2}}{Z^{2}}\right) \log C
$$

where $\gamma$ is a function that is determined by the surface potential $\psi_{o}$,

$$
\gamma=\left[\frac{\exp \left(Z e \psi_{o} / k T\right)-1}{\exp \left(Z E \psi_{o} / k T\right)+1}\right]
$$

Plots of $\log W$ versus $\log C$ are shown in Figure 1.31. The condition $\log W=0$ $(W=1)$ is the onset of fast flocculation, and the electrolyte concentration at this point

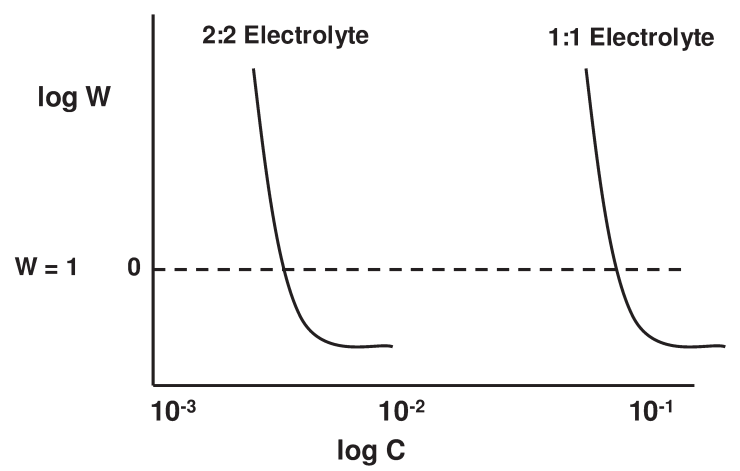

Figure 1.31 Log $W$ versus $\log C$ curves for electrostatically stabilized emulsions. 
defines the critical flocculation concentration (CFC). Above the CFC, $W<1$ (due to the contribution of van der Waals attraction which accelerates the rate above the Smoluchowski value). Below the CFC, $W>1$ and continues to increase with a decrease of the electrolyte concentration. The data in Figure 1.31 also show that the CFC decreases with increase of valency, in accordance to the Schultze-Hardy rule.

Another mechanism of flocculation is that involving the secondary minimum $\left(G_{\text {min }}\right)$ which is few $k T$ units. In this case flocculation is weak and reversible, and hence one must consider both the rate of flocculation (forward rate $k_{f}$ ) and deflocculation (backward rate $k_{b}$ ). The rate of decrease of particle number with time is given by the expression

$$
-\frac{d n}{d t}=-k_{f} n^{2}+k_{b} n
$$

The backward reaction (break-up of weak flocs) reduces the overall rate of flocculation.

\subsubsection{Flocculation of Sterically Stabilized Emulsions}

This occurs when the solvency of the medium for the chain becomes worse than for a $\theta$-solvent $(\chi>0.5)$. Under these conditions, $G_{\text {mix }}$ becomes negative (i.e. attractive) and a deep minimum is produced which results in catastrophic flocculation (referred to as incipient flocculation). This situation is shown schematically in Figure 1.32.

With many systems a good correlation between the flocculation point and the $\theta$ point is obtained. For example, the emulsion will flocculate at a temperature which is referred to as the critical flocculation temperature (CFT) that is equal to the $\theta$-temperature of the stabilizing chain. The emulsion may flocculate at a critical volume fraction of a nonsolvent critical flotation volume (CFV), which is equal to the volume of nonsolvent that brings it to a $\theta$-solvent.

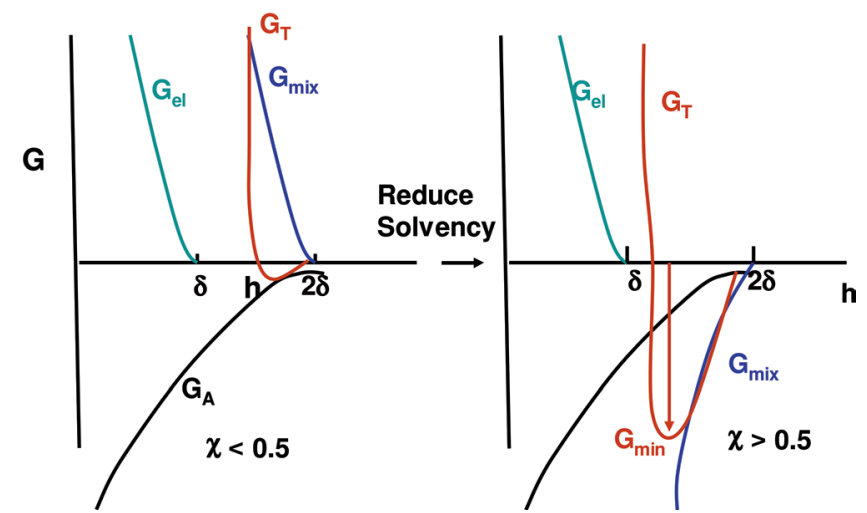

Figure 1.32 Schematic representation of flocculation of sterically stabilized emulsions. 
1.9 .2

\section{General Rules for Reducing (Eliminating) Flocculation}

The rules to be applied for the reduction and/or elimination of flocculation depend on whether charge-stabilized or sterically stabilized emulsions are present.

Charge-Stabilized Emulsions For charge-stabilized emulsions (e.g. using ionic surfactants) the most important criterion is to make $G_{\max }$ as high as possible; this is achieved in three main ways: (i) with a high surface or zeta potential; (ii) with a low electrolyte concentration; and (iii) with a low valency of ions.

Sterically Stabilized Emulsions In the case of sterically stabilized emulsions, four main criteria are necessary:

- Complete coverage of the droplets by the stabilizing chains.

- Firm attachment (strong anchoring) of the chains to the droplets. This requires the chains to be insoluble in the medium and soluble in the oil, but it is incompatible with stabilization which requires a chain to be soluble in the medium yet strongly solvated by its molecules. These conflicting requirements are solved by using A-B, A-B-A block or $\mathrm{BA}_{n}$ graft copolymers (where $\mathrm{B}$ is the 'anchor' chain and $\mathrm{A}$ is the stabilizing chain(s)). Examples of B chains for O/W emulsions are polystyrene, polymethylmethacrylate, polypropylene oxide and alkyl polypropylene oxide. For the A chain(s), PEO or polyvinyl alcohol are good examples. For W/O emulsions, PEO can form the B chain, whereas the Achain(s) maybe polyhydroxy stearic acid (PHS), which is strongly solvated by most oils.

- Thick adsorbed layers; the adsorbed layer thickness should be in the region of $5-10 \mathrm{~nm}$. This means that the molecular weight of the stabilizing chains could be in the region of $1000-5000 \mathrm{Da}$.

- The stabilizing chain should be maintained in good solvent conditions $(\chi<0.5)$, under all conditions of temperature changes on storage.

\subsection{0}

\section{Ostwald Ripening}

The driving force for Ostwald ripening is the difference in solubility between the small and large droplets (the smaller droplets have higher Laplace pressure and higher solubility than their larger counterparts). This is illustrated in Figure 1.33, where $R_{1}$ decreases and $R_{2}$ increases as a result of diffusion of molecules from the smaller to the larger droplets.

The difference in chemical potential between different-sized droplets was first proposed by Lord Kelvin [36]:

$$
S(r)=S(\infty) \exp \left(\frac{2 \gamma V_{m}}{r R T}\right)
$$




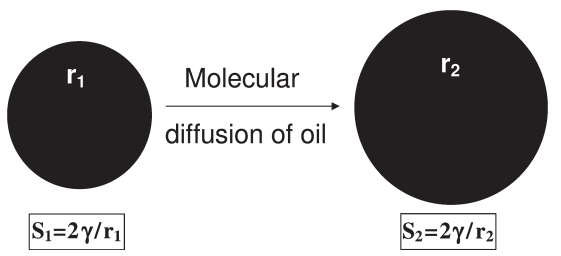

Figure 1.33 Schematic representation of Ostwald ripening.

where $S(r)$ is the solubility surrounding a particle of radius $r, S(\infty)$ is the bulk solubility, $V_{m}$ is the molar volume of the dispersed phase, $R$ is the gas constant and $T$ is the absolute temperature. The quantity $\left(2 \gamma V_{m} / R T\right)$ is termed the characteristic length, and has an order of $\sim 1 \mathrm{~nm}$ or less, indicating that the difference in solubility of a $1 \mu \mathrm{m}$ droplet is of the order of $0.1 \%$, or less. In theory, Ostwald ripening should lead to the condensation of all droplets into a single drop, but this does not occur in practice as the rate of growth decreases with an increase of droplet size.

For two droplets with radii $r_{1}$ and $r_{2}\left(r_{1}<r_{2}\right)$,

$$
\frac{R T}{V_{m}} \ln \left[\frac{S\left(r_{1}\right)}{S\left(r_{2}\right)}\right]=2 \gamma\left[\frac{1}{r_{1}}-\frac{1}{r_{2}}\right]
$$

From Equation (1.90) it can be shown that the larger the difference between $r_{1}$ and $r_{2}$, the higher the rate of Ostwald ripening.

Ostwald ripening can be quantitatively assessed from plots of the cube of the radius versus time $t[37,38]$ :

$$
r^{3}=\frac{8}{9}\left[\frac{S(\infty) \gamma V_{m} D}{\rho R T}\right] t
$$

where $D$ is the diffusion coefficient of the disperse phase in the continuous phase and $\rho$ is the density of the disperse phase.

Several methods may be applied to reduce Ostwald ripening [39-41], including:

- The addition of a second disperse phase component which is insoluble in the continuous medium (e.g. squalane). In this case, partitioning between different droplet sizes occurs, with the component having low solubility expected to be concentrated in the smaller droplets. During Ostwald ripening in a two-component system, equilibrium is established when the difference in chemical potential between different size droplets (which results from curvature effects) is balanced by the difference in chemical potential resulting from partitioning of the two components. This effect reduces further growth of droplets.

- Modification of the interfacial film at the O/W interface. According to Equation 1.91, a reduction in $\gamma$ results in a reduction of the Ostwald ripening rate. By using surfactants that are strongly adsorbed at the O/W interface (i.e. polymeric surfactants) and which do not desorb during ripening (by choosing a molecule that is insoluble in the continuous phase), the rate could be significantly reduced. An increase in the surface dilational modulus $\varepsilon(=\mathrm{d} \gamma / \mathrm{d} \ln A)$ and a decrease in $\gamma$ would be observed for the shrinking drop, and this tends to reduce further growth. 
- A-B-A block copolymers such as PHS-PEO-PHS (which is soluble in the oil droplets but insoluble in water) can be used to achieve the above effect. Similar effects can also be obtained using a graft copolymer of hydrophobically modified inulin, namely INUTEC ${ }^{\circledR}$ SP1 (ORAFTI, Belgium). This polymeric surfactant adsorbs with several alkyl chains (which may dissolve in the oil phase) to leave loops and tails of strongly hydrated inulin (polyfructose) chains. The molecule has limited solubility in water and hence it resides at the O/W interface. These polymeric emulsifiers enhance the Gibbs elasticity, thus significantly reducing the Ostwald ripening rate.

\subsection{1}

\section{Emulsion Coalescence}

When two emulsion droplets come in close contact in a floc or creamed layer, or during Brownian diffusion, a thinning and disruption of the liquid film may occur that results in eventual rupture. On close approach of the droplets, film thickness fluctuations may occur; alternatively, the liquid surfaces undergo some fluctuations forming surface waves, as illustrated in Figure 1.34.

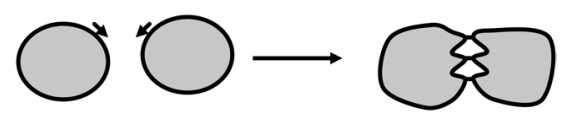

Figure 1.34 Schematic representation of surface fluctuations.

The surface waves may grow in amplitude and the apices may join as a result of the strong van der Waals attraction (at the apex, the film thickness is the smallest). The same applies if the film thins to a small value (critical thickness for coalescence).

A very useful concept was introduced by Deryaguin and Scherbaker [42], who suggested that a 'disjoining pressure' $\pi(h)$ is produced in the film which balances the excess normal pressure,

$$
\pi(h)=P(h)-P_{o}
$$

where $P(h)$ is the pressure of a film with thickness $h$, and $P_{o}$ is the pressure of a sufficiently thick film such that the net interaction free energy is zero.

$\pi(h)$ may be equated to the net force (or energy) per unit area acting across the film:

$$
\pi(h)=-\frac{d G_{T}}{d h}
$$

where $G_{T}$ is the total interaction energy in the film.

$\pi(h)$ is composed of three contributions due to electrostatic repulsion $\left(\pi_{E}\right)$, steric repulsion $\left(\pi_{s}\right)$ and van der Waals attraction $\left(\pi_{A}\right)$ :

$$
\pi(h)=\pi_{E}+\pi_{s}+\pi_{A}
$$




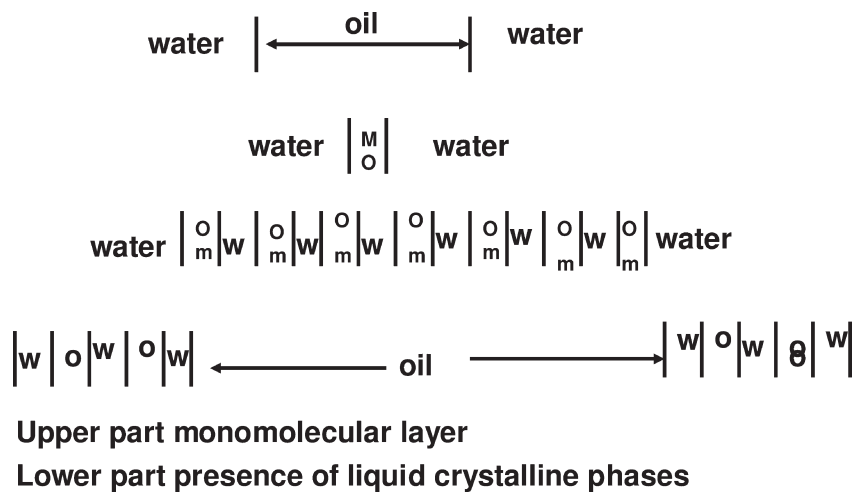

Figure 1.35 Schematic representation of the role of liquid crystalline phases.

In order to produce a stable film $\pi_{E}+\pi_{s}>\pi_{A}$, and this is the driving force for the prevention of coalescence that may be achieved by either of two mechanisms (and/or their combination):

- Increased repulsion, both electrostatic and steric.

- Dampening of the fluctuation by enhancing the Gibbs elasticity. In general, smaller droplets are less susceptible to surface fluctuations and hence coalescence is reduced. This explains the high stability of nanoemulsions.

Several methods may be applied to achieve the above effects:

- The use of mixed surfactant films: In many cases the use of mixed surfactants (e.g. anionic and nonionic or long-chain alcohols) can reduce coalescence as a result of various effects such as high Gibbs elasticity, high surface viscosity, and hindered diffusion of the surfactant molecules from the film.

- The formation of lamellar liquid crystalline phases at the O/W interface: This mechanism was suggested by Friberg and coworkers [43], who proposed that the surfactant or mixed surfactant film could produce several bilayers that 'wrapped' the droplets. As a result of these multilayer structures, the potential drop is shifted to longer distances, thus reducing the van der Waals attraction. A schematic representation of the role of liquid crystals is shown in Figure 1.35, which shows the difference between having a monomolecular layer and a multilayer, as is the case with liquid crystals. In order for coalescence to occur, these multilayers must be removed 'two-by-two', and this forms an energy barrier that prevents coalescence.

\subsection{1}

Rate of Coalescence

Since film drainage and rupture is a kinetic process, coalescence is also a kinetic process. If the number of particles $n$ (flocculated or not) is measured at time $t$, then 


$$
n=n_{t}+n_{v} m
$$

where $n_{t}$ is the number of primary particles remaining, and $n$ is the number of aggregates consisting of $m$ separate particles. When studying emulsion coalescence, it is important to consider the rate constant of flocculation and coalescence; if coalescence is the dominant factor, then the rate $K$ follows a first-order kinetics:

$$
n=\frac{n_{0}}{K t}[1+\exp (-K t)]
$$

Hence, a plot of $\log n$ versus $t$ should give a straight line from which $K$ can be calculated.

\subsection{1 .2}

\section{Phase Inversion}

The phase inversion of emulsions can be one of two types: (i) Transitional inversion, which is induced by changing the facers which affect the HLB of the system (e.g. temperature and/or electrolyte concentration); and (ii) catastrophic inversion, which is induced by increasing the volume fraction of the disperse phase.

Catastrophic inversion is illustrated graphically in Figure 1.36, which shows the variation of viscosity and conductivity with the oil volume fraction $\phi$. It can be seen that inversion occurs at a critical $\phi$, which may be identified with the maximum packing fraction. At $\phi_{c r} \eta$ suddenly decreases, such that the inverted W/O emulsion has a much lower volume fraction. $\kappa$ also decreases sharply at the inversion point as the continuous phase is now oil.

Earlier theories of phase inversion were based on packing parameters. When $\phi$ exceeds the maximum packing ( $\sim 0.64$ for random packing and $\sim 0.74$ for hexagonal packing of monodisperse spheres; for polydisperse systems, the maximum packing exceeds 0.74 ), inversion occurs. However, these theories are not adequate, as many emulsions invert at $\varphi$-values well below the maximum packing as a result of changes in surfactant characteristics with variation of conditions. For example, when using a

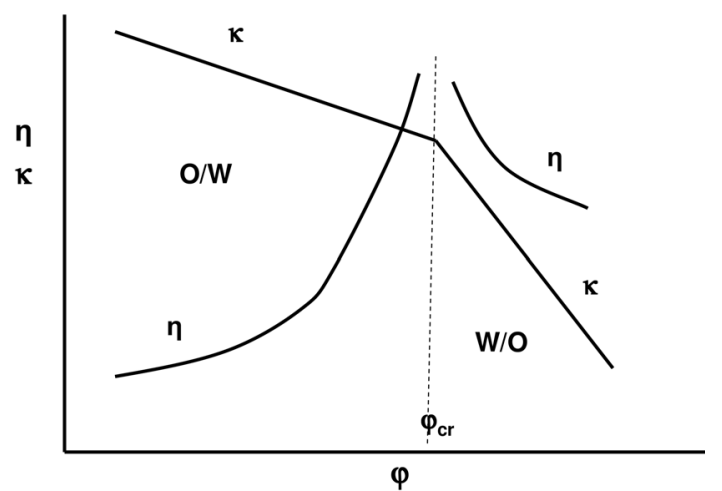

Figure 1.36 Variation of conductivity $(\kappa)$ and viscosity $(\eta)$ with the volume fraction of oil, $\phi$. 
nonionic surfactant based on PEO, the latter chain changes its solvation by an increase of temperature and/or addition of electrolyte. Many emulsions show phase inversion at a critical temperature (the phase inversion temperature) that depends on the HLB number of the surfactant as well as the presence of electrolytes. By increasing the temperature and/or addition of electrolyte, the PEO chains become dehydrated and finally more soluble in the oil phase. Under these conditions, the $\mathrm{O} / \mathrm{W}$ emulsion will invert to a $\mathrm{W} / \mathrm{O}$ emulsion. The above dehydration effect amounts to a decrease in the HLB number, and when the latter reaches a value that is more suitable for W/O emulsion, then inversion will occur. At present, there is no quantitative theory that accounts for the phase inversion of emulsions.

\subsection{2}

\section{Rheology of Emulsions}

Although the rheology of emulsions has many similar features to that of suspensions, there are three main differences in aspect:

- The mobile liquid/liquid interface that contains surfactant or polymer layers introduces a response to deformation; hence, the interfacial rheology must be considered.

- The dispersed phase viscosity relative to that of the medium has an effect on the rheology of the emulsion.

- The deformable nature of the disperse phase droplets, particularly for large droplets, has an effect on the emulsion rheology at high phase volume fraction $\phi$.

When the above factors are considered, the bulk rheology of emulsions can be treated in a similar manner as for suspensions, and the same techniques applied.

\subsection{1}

\section{Interfacial Rheology}

A fluid interface in equilibrium exhibits an intrinsic state of tension that is characterized by its interfacial tension $\gamma$ which is given by the change in free energy with area of the interface, at constant composition $n_{i}$ and temperature $T$,

$$
\gamma=\left(\frac{\partial G}{\partial A}\right)_{n_{i}, T}
$$

The unit for $\gamma$ is energy per unit area $\left(\mathrm{mJ} \mathrm{m}^{-1}\right)$ or force per unit length $\left(\mathrm{mN} \mathrm{m}^{-1}\right)$, which are dimensionally equivalent.

The adsorption of surfactants or polymers lowers the interfacial tension, and this produces a two-dimensional surface pressure $\pi$ that is given by

$$
\pi=\gamma_{0}-\gamma
$$

where $\gamma_{o}$ is the interfacial tension of the 'clean' interface (before adsorption) and $\gamma$ that after adsorption. 
The interface is considered to be a macroscopically planar, dynamic fluid interface. Thus, the interface is regarded as a two-dimensional entity independent of the surrounding three-dimensional fluid. The interface is considered to correspond to a highly viscous insoluble monolayer and the interfacial stress $\sigma_{s}$ acting within such a monolayer is sufficiently large compared to the bulk-fluid stress acting across the interface. In this way it is possible to define an interfacial shear viscosity $\eta_{s}$,

$$
\sigma_{s}=\eta_{s} \gamma
$$

where $\gamma$ is the shear rate, $\eta_{s}$ is given in surface $\mathrm{Pa} \cdot \mathrm{s}\left(\mathrm{Nm}^{-1} \mathrm{~s}\right)$ or surface poise (dyne $\mathrm{cm}^{-1} \mathrm{~s}$ ). At this point it should be noted that the surface viscosity of a surfactant-free interface is negligible and can reach high values for adsorbed rigid molecules such as proteins.

\subsection{2 .2}

\section{Measurement of Interfacial Viscosity}

Many surface viscometers utilize torsional stress measurements upon rotating a ring, disk or knife edge (shown schematically in Figure 1.37) within or near to the liquid/liquid interface [44]. This type of viscometer is moderately sensitive. For a disk viscometer the interfacial shear viscosity can be measured in the range $\eta_{s} \geq 10^{-2}$ surface $\mathrm{Pa} \cdot \mathrm{s}$. The disk is rotated within the plane of the interface with angular velocity $\omega$. A torque is then exerted on the disk of radius $R$ by both the surfactant film with surface viscosity $\eta_{s}$ and the viscous liquid (with bulk viscosity $\eta$ ) that is given by the expression

$$
M=(8 / 3) R^{3} \eta \omega+4 \pi R^{2} \eta_{s} \omega
$$

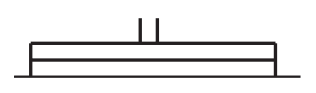

Ring viscometer

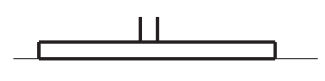

\section{Disk viscometer}

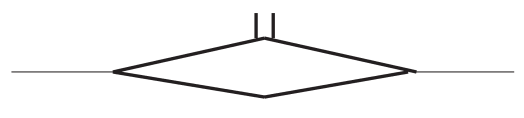

\section{Knife-edge viscometer}

Figure 1.37 Schematic representation of surface viscometers.

\subsection{2 .3}

\section{Interfacial Dilational Elasticity}

The interfacial dilational (Gibbs) elasticity $\varepsilon$, which is an important parameter in determining emulsion stability (reduction of coalescence during formation), is given by the equation: 


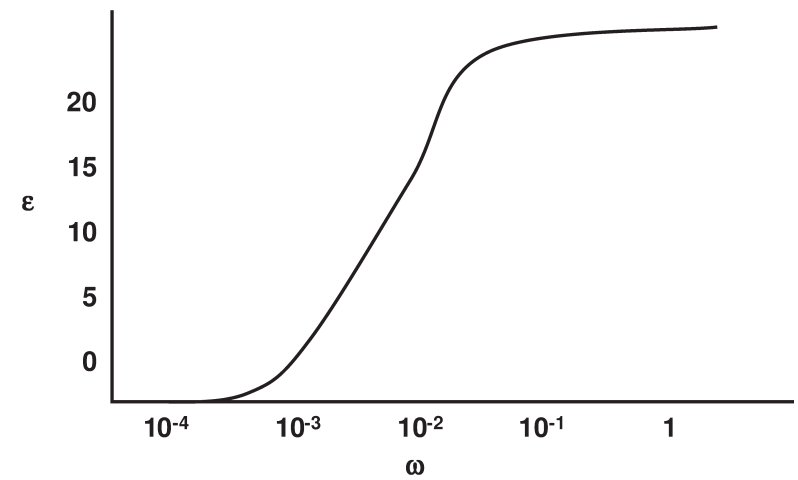

Figure 1.38 Gibbs dilational elasticity versus frequency.

$$
\varepsilon=\frac{d \gamma}{d \ln A}
$$

where $d \gamma$ is the change in interfacial tension during expansion of the interface by an amount $d A$ (referred to as interfacial tension gradient resulting from nonuniform surfactant adsorption on expansion of the interface).

One of the most convenient methods for measuring $\varepsilon$ is to use a Langmuir trough with two moving barriers for expansion and compression of the interface. Another method is to use the oscillating bubble technique, for which instruments are commercially available. A further useful technique for measuring $\varepsilon$ is the 'pulsed drop method'. Here, a rapid expansion of a droplet at the end of a capillary from radius $r_{1}$ to $r_{2}$ is obtained by the application of pressure. The pressure drop within the droplet is measured as a function of time using a sensitive pressure transducer, and from this it is possible to obtain the interfacial tension as a function of time. The Gibbs dilational elasticity is determined from values of the time-dependent interfacial tension. Measurement can be made as a function of frequency, as shown in Figure 1.38 for stearic acid at the decane-water interface at $\mathrm{pH} 2.5$.

\subsection{2 .4}

\section{Interfacial Dilational Viscosity}

Measurement of the dilational viscosity is more difficult than that of the interfacial shear viscosity, due mainly to the coupling between dilational viscous and elastic components. The most convenient method for measuring dilational viscosity is the maximum bubble pressure technique, that can be only applied at the air/water interface [45]. According to this technique, the pressure drop across the bubble surface at the instant when the bubble possesses a hemispherical shape (corresponding to the maximum pressure) is due to a combination of bulk viscous, surface tension and surface dilational viscosity effects; this allows the interfacial dilational viscosity to be obtained. 


\subsection{5}

\section{Non-Newtonian Effects}

Most adsorbed surfactant and polymer coils at the O/W interface show nonNewtonian rheological behavior. The surface shear viscosity $\eta_{s}$ depends on the applied shear rate, showing shear thinning at high shear rates. Some films also show Bingham plastic behavior with a measurable yield stress. As many adsorbed polymers and proteins demonstrate viscoelastic behavior, it is possible to measure viscous and elastic components using sinusoidally oscillating surface dilation. For example, the complex dilational modulus $\varepsilon^{*}$ which is obtained can be split into 'in-phase' (the elastic component $\varepsilon^{\prime}$ ) and 'out-of-phase' (the viscous component $\varepsilon^{\prime \prime}$ ) components. Creep and stress relaxation methods can be applied to study viscoelasticity.

\subsection{2 .6}

\section{Correlation of Interfacial Rheology with Emulsion Stability}

\subsubsection{Mixed Surfactant Films}

Prince et al. [46] found that emulsions prepared using a mixture of SDS and dodecyl alcohol are more stable than those prepared using SDS alone. This enhanced stability is due to the higher interfacial dilational elasticity $\varepsilon$ for the mixture when compared to that of SDS alone. Interfacial dilational viscosity did not play a major role as the emulsions are stable at high temperature, whereby the interfacial viscosity becomes lower.

The above correlation is not general for all surfactant films, since other factors such as thinning of the film between emulsion droplets (which depends on other factors such as repulsive forces) can also play a major role.

\subsubsection{Protein Films}

Biswas and Haydon [47] identified some correlation between the viscoelastic properties of protein (albumin or arabinic acid) films at the $\mathrm{O} / \mathrm{W}$ interface and the stability of emulsion drops against coalescence. Viscoelastic measurements were carried out using creep and stress relaxation measurements (using a specially designed interfacial rheometer). A constant torque or stress $\sigma\left(\mathrm{mN} \mathrm{m}^{-1}\right)$ was applied and the deformation $\gamma$ measured as a function of time for $30 \mathrm{~min}$. After this period the torque was removed and $\gamma$ (which changes sign) was measured as a function of time to obtain the recovery curve. The results are illustrated graphically in Figure 1.39.

From the creep curves it is possible to obtain the instantaneous modulus $G_{o}(\sigma /$ $\gamma_{\text {inst. }}$ ) and the surface viscosity $\eta_{s}$ from the slope of the straight line (which gives the shear rate) and the applied stress. $G_{o}$ and $\eta_{s}$ are plotted versus $\mathrm{pH}$, as shown in Figure 1.40. Both show an increased creep with increased $\mathrm{pH}$, reaching a maximum at $\mathrm{pH} \sim 6$ (the isoelectric point of the protein), when the protein molecules show maximum rigidity at the interface.

The stability of the emulsion was assessed by measuring the residence time $(t)$ of several oil droplets at a planar $\mathrm{O} / \mathrm{W}$ interface containing the adsorbed protein. Figure 1.40 shows the variation of $t_{1 / 2}$ (the time taken for half the number of oil 


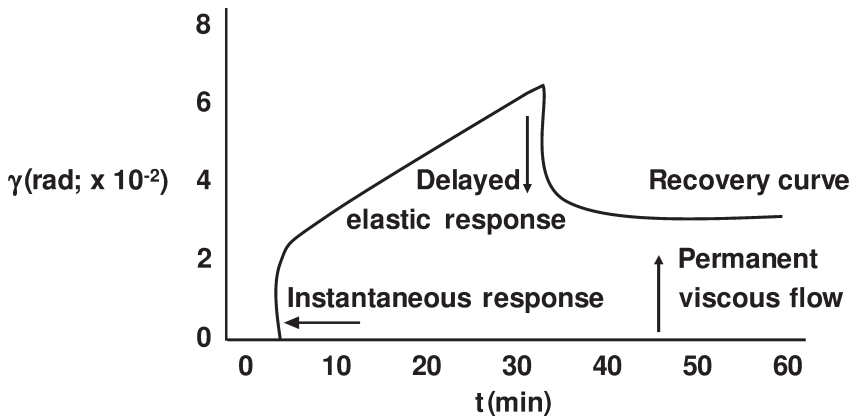

Figure 1.39 Creep curve for a protein film at the oil/water $(\mathrm{O} / \mathrm{W})$ interface.

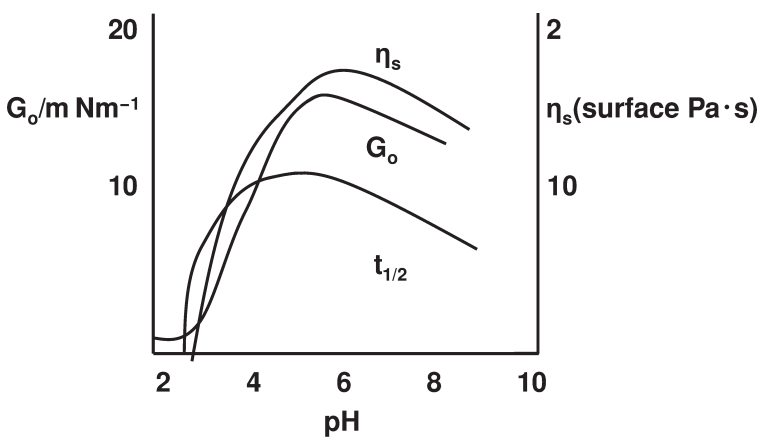

Figure 1.40 Variation of $t_{1 / 2}$ and $G_{o}$ and $\eta_{s}$ with $\mathrm{pH}$.

droplets to coalesce with the oil at the $\mathrm{O} / \mathrm{W}$ interface) with $\mathrm{pH}$. A good correlation between $t_{1 / 2}$ and $G_{o}$ and $\eta_{s}$ was obtained.

Biswas and Haydon subsequently derived a relationship between coalescence time $\tau$ and surface viscosity $\eta_{s}$, instantaneous modulus $G_{o}$ and adsorbed film thickness $h$ :

$$
\tau=\eta_{s}\left[3 C^{\prime} \frac{h^{2}}{A}-\frac{1}{G_{o}}-\phi(t)\right]
$$

where $3 C^{\prime}$ is a critical deformation factor, $A$ is the Hamaker constant and $\phi(t)$ is the elastic deformation per unit stress.

Equation 1.102 shows that (increases with increase of $\eta_{s}$, but most importantly it is directly proportional to $h_{2}$. These results show that viscoelasticity is necessary, but not sufficient, to ensure stability against coalescence. In order to ensure the stability of an emulsion it must be ensured that $h$ is large enough and film drainage is prevented.

\subsection{7}

\section{Bulk Rheology of Emulsions}

For rigid (highly viscous) oil droplets dispersed in a medium of low viscosity such as water, the relative viscosity $\eta_{r}$ of a dilute (volume fraction $\phi \leq 0.01$ ) O/W emulsion of noninteracting droplets behaves as 'hard-spheres' (similar to suspensions). 
In the above case, $\eta_{r}$ is given by the Einstein equation,

$$
\eta_{r}=1+[\eta] \phi
$$

where $[\eta]$ is the intrinsic viscosity that is equal to 2.5 for hard spheres.

For droplets with low viscosity (comparable to that of the medium), the transmission of tangential stress across the $\mathrm{O} / \mathrm{W}$ interface, from the continuous phase to the dispersed phase, causes liquid circulation in the droplets. Energy dissipation is less than that for hard spheres, and the relative viscosity is lower than that predicted by the Einstein equation [48-51].

For an emulsion with viscosity $\eta_{i}$ for the disperse phase and $\eta_{o}$ for the continuous phase

$$
[\eta]=2.5\left(\frac{\eta_{i}+0.4 \eta_{o}}{\eta_{i}+\eta_{o}}\right)
$$

Clearly when $\eta_{i} \gg \eta_{o}$, the droplets behave as rigid spheres and [ $\left.\eta\right]$ approaches the Einstein limit of 2.5. In contrast if $\eta_{i} \ll \eta_{o}$ (as is the case for foams), $[\eta]=1$.

In the presence of viscous interfacial layers, Equation 1.104 is modified to take into account the surface shear viscosity $\eta_{s}$ and surface dilational viscosity $\mu_{s}$ :

$$
\begin{aligned}
& {[\eta]=2.5\left(\frac{\eta_{i}+0.4 \eta_{o}+\xi}{\eta_{i}+\eta_{o}+\xi}\right)} \\
& \xi=\frac{\left(2 \eta_{s}+3 \mu_{s}\right)}{R}
\end{aligned}
$$

where $R$ is the droplet radius.

\subsection{2 .8}

\section{Rheology of Concentrated Emulsions}

When the volume fraction of droplets exceed the Einstein limit (i.e. $\phi>0.01$ ), it is essential to take into account the effect of Brownian motion and interparticle interactions. The smaller the emulsion droplets, the more important the contribution of Brownian motion and colloidal interactions. Brownian diffusion tends to randomize the position of colloidal particles, leading to the formation of temporary doublets, triplets, and so on. The hydrodynamic interactions are of longer range than the colloidal interactions, and they come into play at relatively low volume fractions $(\phi>0.01)$; this results in an ordering of the particles into layers and tends to destroy the temporary aggregates caused by the Brownian diffusion. This explains the shear thinning behavior of emulsions at high shear rates.

For the volume fraction range $0.01<\phi<0.2$, Batchelor derived the following expression for a dispersion of hydrodynamically interacting hard spheres:

$$
\eta_{r}=1+2.5 \phi+6.2 \phi^{2}+\vartheta \phi^{3}
$$




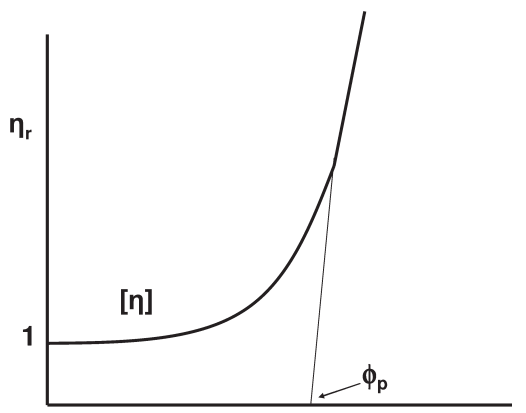

\section{$\phi$}

Figure 1.41 The $\eta_{r}-\phi$ curve.

The second term in Equation 1.107 is the Einstein limit, the third term accounts for the hydrodynamic (two-body) interaction, while the fourth term relates to multibody interaction.

At higher volume fractions $(\phi>0.2), \eta_{r}$ is a complex function of $\phi$, and the $\eta_{r}-\phi$ curve is shown in Figure 1.41. This curve is characterized by two asymptotes, namely $[\eta]$ the intrinsic viscosity and $\phi_{p}$. A good semi-empirical equation that fits the curve has been provided by Dougherty and Krieger:

$$
\eta_{r}=\left(1-\frac{\phi}{\phi_{p}}\right)^{-[\eta] \phi_{p}}
$$

Experimental results of $\eta_{r}-\phi$ curves were obtained for paraffin $\mathrm{O} / \mathrm{W}$ emulsions stabilized with an A-B-C surfactant consisting of nonyl phenol (B), 13 moles propylene oxide (C) and PEO with 27, 48, 80 and 174 moles EO. As an illustration, Figure 1.42 shows the results for an emulsion stabilized with the surfactant

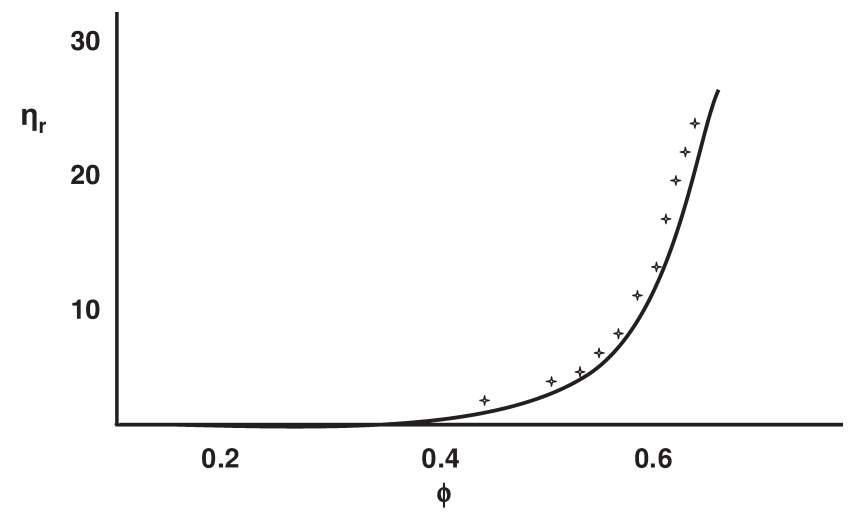

\section{+ Experimental results}

Full line calculated curve using Dougherty-Krieger equation

Figure 1.42 Experimental and theoretical $\eta_{r}-\phi$ curves. 
containing 27 moles EO (the volume medium diameter of the droplets is $3.5(\mu \mathrm{m})$. The calculations based on the Dougherty-Krieger equation are also shown in the same figure. In these calculations $[\eta]=2.5$ and $\phi_{p}$ was obtained from a plot of $\eta^{-1 / 2}$ versus $\phi$ and extrapolation of the straight line to $\eta^{-1 / 2}=0$. The value of $\phi_{p}$ was 0.73 (which is higher than the maximum random packing of 0.64 , as a result of the polydispersity of the emulsion). The results using the other three surfactants showed the same trend; the experimental $\eta_{r}-\phi$ curves were close to those calculated using the Dougherty-Krieger equation, indicating that these emulsions were behaving as hard spheres.

\subsection{2 .9}

\section{Influence of Droplet Deformability on Emulsion Rheology}

The influence of droplet deformability on emulsion rheology was investigated by comparing the $\eta_{r}-\phi$ curves of hard spheres of silica with two polydimethylsiloxane (PDMS) emulsions with low (PDMS 0.3) and high deformability (PDMS 0.45) (by controlling the proportion of crosslinking agent for the droplets; 0.3 low and 0.45 high crosslinking agent). The $\eta_{r}-\phi$ curves for the three systems are shown in Figure 1.43. The $\eta_{r}-\phi$ curve for silica can be fitted by the Dougherty-Krieger equation over the whole volume fraction range, indicating typical hard-sphere behavior. The $\eta_{r}-\phi$ curve for the less deformable PDMS deviates from the hardsphere curve at $\phi=0.58$. The $\eta_{r}-\phi$ curve for the more deformable PDMS deviates from the hard-sphere curve at $\phi=0.40$, clearly showing the deformation of the 'soft' droplets at a relatively low volume fraction.

\subsubsection{0}

\section{Viscoelastic Properties of Concentrated Emulsions}

The viscoelastic properties of emulsions can be investigated using dynamic (oscillatory) measurements $[49,50]$. A sinusoidal strain with amplitude $\gamma_{o}$ is applied to the system at a frequency $\omega\left(\mathrm{rad} \mathrm{s}^{-1}\right)$ and the stress $\sigma$ (with amplitude $\sigma_{o}$ ) is simultaneously measured. From the time shift $\Delta t$ between the sine waves of strain and stress

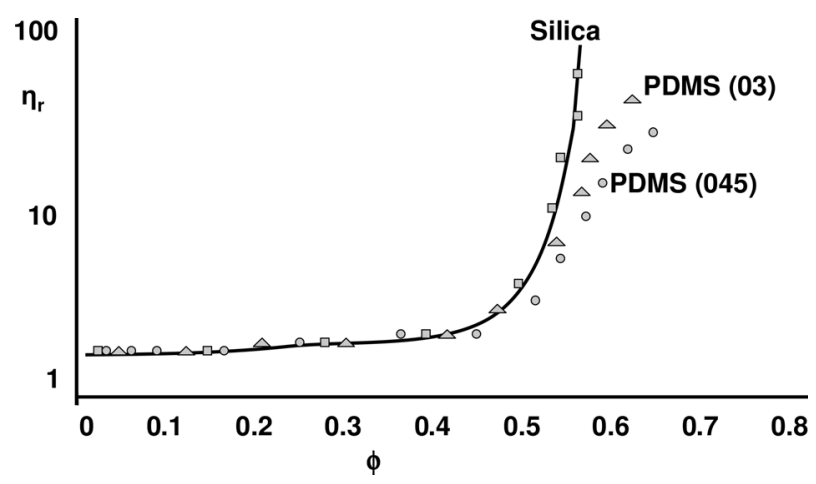

Figure 1.43 $\eta_{r}-\phi$ curves for silica and two PDMS emulsions. 


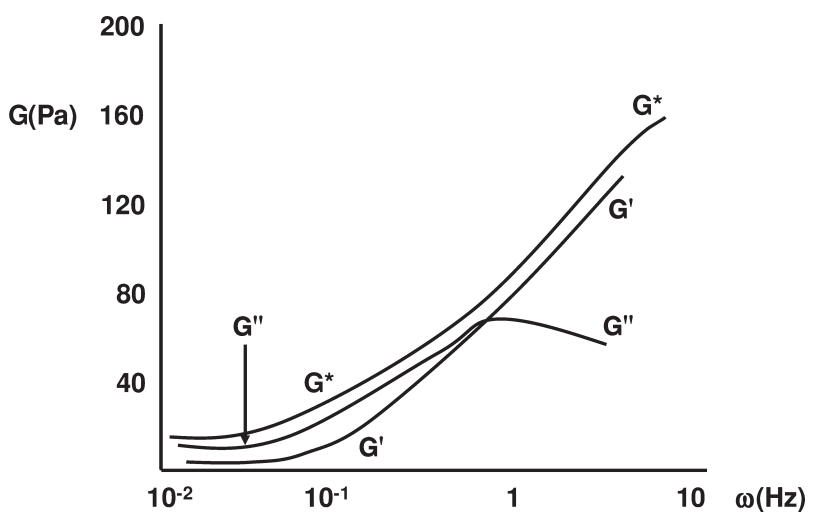

Figure 1.44 Variation of $G^{*}, G^{\prime}$ and $G^{\prime \prime}$ with frequency $\omega$.

one can measure the phase angle shift $\delta(\delta=\Delta t \omega)$. From $\sigma_{o}, \gamma_{o}$ and $\delta$ it is then possible to obtain the complex modulus $G^{*}$, the storage modulus $G^{\prime}$ (the elastic component) and the loss modulus $G^{\prime \prime}$ (the viscous component).

$G^{*}, G^{\prime}$ and $G^{\prime \prime}$ are measured as a function of strain amplitude to obtain the linear viscoelastic region, and then as a function of frequency (keeping $\gamma_{o}$ in the linear region). As an illustration, Figure 1.44 shows the results for an $\mathrm{O} / \mathrm{W}$ emulsion at $\phi=0.6$ (the emulsion was prepared using an A-B-A block copolymer of PEO (A) and polypropylene oxide (PPO, B) with an average of $47 \mathrm{PO}$ units and $42 \mathrm{EO}$ units.

The data shown in Figure 1.44 are typical for a viscoelastic liquid. In the lowfrequency regime $(<1 \mathrm{~Hz}), G^{\prime \prime}>G^{\prime}$, but as the frequency $\omega$ increases so too does $G^{\prime}$ increase. At a characteristic frequency $\omega^{*}$ (the crossover point) $G^{\prime}$ becomes higher than $G^{\prime \prime}$, and at high frequency it becomes closer to $G^{*}$. $G^{\prime \prime}$ then increases with the rise in frequency to reach a maximum at $\omega^{*}$, after which it decreases with any further increase in frequency.

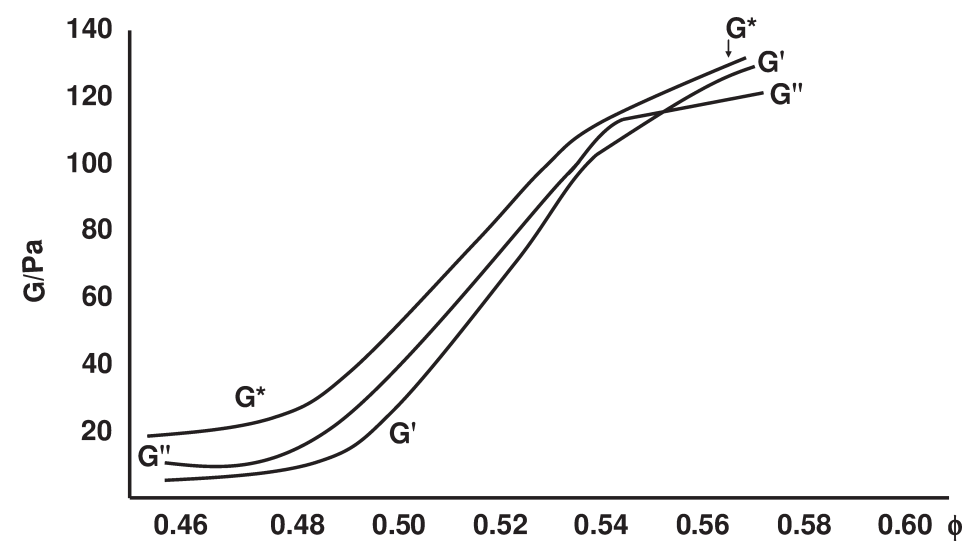

Figure 1.45 Variation of $G^{*}, G^{\prime}$ and $G^{\prime \prime}$ with $\phi$. 
From $\omega^{*}$, the relaxation time $t^{*}$ can be calculated:

$$
t^{*}=\frac{1}{2 \pi \omega^{*}}
$$

For the above value of $\phi(=0.6), t^{*}=0.12 \mathrm{~s}$. In fact, $t^{*}$ was seen to increase with an increase of $\phi$, and this reflected the stronger interaction with an increase of $\phi$.

In order to obtain the onset of strong elastic interaction in emulsions, $G^{*}, G^{\prime}$ and $G^{\prime \prime}$ (obtained in the linear viscoelastic region and high frequency, e.g. $1 \mathrm{~Hz}$ ) are plotted versus the volume fraction of the emulsion $\phi$. Care must be taken to ensure that the droplet size distribution in all emulsions is the same. The most convenient way to do this is to prepare an emulsion at the highest possible $\phi$ (e.g. 0.6), and then dilute this to obtain a range of $\phi$-values. Droplet size analysis should be obtained for each emulsion to ensure that the size distribution is the same. Figure 1.45 shows the plots for $G^{*}, G^{\prime}$ and $G^{\prime \prime}$ versus $\phi$. At $\phi<0.56, G^{\prime \prime}>G^{\prime}$, whereas at $\phi>0.56, G^{\prime}>G$; $\phi=0.56$ is the onset of predominantly elastic interaction, and this reflects the small distance of separation between the droplets.

\section{References}

1 Tadros, Th.F. and Vincent, B. (1983) in Encyclopedia of Emulsion Technology (ed. Becher, P.), Marcel Dekker, New York.

2 Binks, B.P. (ed.) (1998) Modern Aspects of Emulsion Science, The Royal Society of Chemistry Publication.

3 Tadros, Th.F. (2005) Applied Surfactants, Wiley-VCH, Weinheim.

4 Hamaker, H.C. (1937) Physica (Utrecht), 4, 1058.

5 Deryaguin, B.V. and Landua, L. (1941) Acta Physicochem. USSR, 14, 633.

6 Verwey, E.J.W. and Overbeek, J.Th.G. (1948) Theory of Stability of Lyophobic Colloids, Elsevier, Amsterdam.

7 Napper, D.H. (1983) Polymeric Stabilisation of Dispersions, Academic Press, London.

8 Walstra, P. and Smolders, P.E.A. (1998) in Modern Aspects of Emulsions (ed. Binks, B.P.), The Royal Society of Chemistry, Cambridge.

9 Stone, H.A. (1994) Annu. Rev. Fluid Mech., 226, 95.

10 Wierenga, J.A., ven Dieren, F., Janssen, J.J.M. and Agterof, W.G.M. (1996) Trans. Inst. Chem. Eng., 74-A, 554.
11 Levich, V.G. (1962) Physicochemical Hydrodynamics, Prentice-Hall, Englewood Cliffs.

12 Davis, J.T. (1972) Turbulent Phenomena, Academic Press, London.

13 Lucasses-Reynders, E.H. (1996) in Encyclopedia of Emulsion Technology (ed. Becher, P.), Marcel Dekker, New York.

14 Graham, D.E. and Phillips, M.C. (1979) J. Colloid Interface Sci., 70, 415.

15 Lucasses-Reynders, E.H. (1994) Colloids and Surfaces, A91, 79.

16 Lucassen, J. (1981) in Anionic Surfactants (ed. E.H. Lucassen-Reynders), Marcel Dekker, New York.

17 van den Tempel, M. (1960) Proceedings of the International Congress on, Surface Activity, 2, 573.

18 (a) Griffin, W.C. (1949) J. Cosmet. Chemists, 1, 311; (b) Griffin, W.C. (1954) J. Cosmet. Chemists, 5, 249.

19 Davies, J.T. (1959) Proceedings of the International Congress on, Surface Activity, $1,426$.

20 Davies, J.T. and Rideal, E.K. (1961) Interfacial Phenomena, Academic Press, New York. 
21 Shinoda, K. (1967) J. Colloid Interface Sci., 25, 396.

22 Shinoda, K. and Saito, H. (1969) J. Colloid Interface Sci., 30, 258.

23 Beerbower, A. and Hill, M.W. (1972) Amer. Cosmet. Perfum., 87, 85.

24 Hildebrand, J.H. (1936) Solubility of NonElectrolytes, 2nd ed. Reinhold, New York.

25 Hansen, C.M. (1967) J. Paint Technol., 39, 505.

26 Barton, A.F.M. (1983) Handbook of Solubility Parameters and Other Cohesive Parameters, CRC Press, New York.

27 Israelachvili, J.N., Mitchell, J.N. and Ninham, B.W. (1976) J. Chem. Soc., Faraday Trans. II, 72, 1525.

28 Tadros, Th.F. (1967) in Solid/Liquid Dispersions (ed. Th.F. Tadros), Academic Press, London.

29 Batchelor, G.K. (1972) J. Fluid. Mech., 52, 245

30 Buscall, R., Goodwin, J.W., Ottewill, R.H. and Tadros, Th.F. (1982) J. Colloid Interface Sci., 85, 78 .

31 Krieger, I.M. (1972) Adv. Colloid Interface Sci., 3, 111.

32 (a) Asakura, S. and Osawa, F. (1954) J. Phys. Chem., 22, 1255; (b) Asakura, S. and Osawa, F. (1958) J. Polym. Sci., 33, 183.

33 Smoluchowski, M.V. (1927) Z. Phys. Chem. 92, 129.

34 Fuchs N. (1936) Z. Physik, 89736.

35 Reerink, H. and Overbeek, J.Th.G. (1954) Disc. Faraday Soc., 18, 74.

36 Thompson, W. (1871) (Lord Kelvin), Philosophical Magazine, 42, 448.

37 Lifshitz I.M. and Slesov V.V. (1959) Soviet Physics JETP, 35, 331.

38 Wagner C. (1961) Z. Electrochem., 35, 581.

39 Kabalanov, A.S. and Shchukin, E.D. (1992) Adv. Colloid Interface Sci., 38, 69.
40 Kabalanov, A.S. (1994) Langmuir, 10, 680.

41 Weers, J.G. (1998) in Modern Aspects of Emulsion Science (ed. Binks, B.P.), Royal Society of Chemistry Publication Cambridge.

42 Deryaguin, B.V. and Scherbaker, R.L. (1961) Kolloidn Zhurnal, 23, 33.

43 Friberg, S., Jansson, P.O. and Cederberg, E. (1976) J. Colloid Interface Sci., 55, 614.

44 Criddle, D.W. (1960) in The viscosity and viscoelasticity of interfaces (ed. Eirich, F.R.), in Rheology, Academic Press, New York, Vol. 3, Chapter 11.

45 Edwards, D.A., Brenner, H. and Wasan, D.T. (1991) Interfacial Transport Processes and Rheology, Butterworth-Heinemann, Boston, London.

46 Prince, A., Arcuri, C. and van den Tempel, M. (1967) J. Colloid and Interface Sci., 24, 811.

47 (a) Biswas, B. and Haydon, D.A. (1963) Proc. Roy. Soc., A271, 296; (b) Biswas, B. and Haydon, D.A. (1963) Proc. Roy. Soc., A271, 317; (c) Biswas, B. and Haydon, D.A. (1962) Kolloidn Zhurnal, 185, 31; (d) Biswas, B. and Haydon, D.A. (1962) Kolloidn Zhurnal, 186, 57.

48 Sherman, P. (1968) Rheology of emulsions (ed. Sherman, P.), in Emulsion Science, Academic Press, London, Chapter 4.

49 Tadros, Th.F. (1991) Rheological properties of emulsion systems (ed. Sjoblom, J.), in Emulsions - A Fundamental and Practical Approach, NATO ASI Series, Vol. 363, Kluwer Academic Publishers, London.

50 Tadros, Th.F. (1994) Colloids and Surfaces A91, 215.

51 Dickinson, E. (1998) Rheology of emulsions (ed. Binks, B.P.), in Modern Aspects of Emulsion Science, Royal Society of Chemistry Publications, Cambridge. 